\title{
A reformulation of strain gradient plasticity
}

\author{
N.A. Fleck ${ }^{\mathrm{a}}$, J.W. Hutchinson ${ }^{\mathrm{b}, *}$ \\ ${ }^{a}$ Engineering Department, Cambridge University, Cambridge, UK \\ ${ }^{\mathrm{b}}$ Division of Engineering and Applied Science, Harvard University, 315 Pierce Hall, 29 Oxford Street, \\ Cambridge, MA 02138, USA
}

Received 13 January 2001; received in revised form 22 March 2001

\begin{abstract}
A class of phenomenological strain gradient plasticity theories is formulated to accommodate more than one material length parameter. The objective is a generalization of the classical $J_{2}$ flow theory of plasticity to account for strain gradient effects that emerge in deformation phenomena at the micron scale. A special case involves a single length parameter and is of similar form to that proposed by Aifantis and co-workers. Distinct computational advantages are associated with this class of theories that make them attractive for applications requiring the generation of numerical solutions. The higher-order nature of the theories is emphasized, involving both higher-order stresses and additional boundary conditions. Competing members in the class of theories will be examined in light of experimental data on wire torsion, sheet bending, indentation and other micron scale plasticity phenomena. The data strongly suggest that at least two distinct material length parameters must be introduced in any phenomenological gradient plasticity theory, one parameter characterizing problems for which stretch gradients are dominant and the other relevant to problems when rotation gradients (or shearing gradients) are controlling. Flow and deformation theory versions of the theory are highlighted that can accommodate multiple length parameters. Examination of several basic problems reveals that the new formulations predict quantitatively similar plastic behavior to the theory proposed earlier by the present authors. The new formulations improve on the earlier theory in the manner in which elastic and plastic strains are decomposed and in the representation of behavior in the elastic range. (C) 2001 Elsevier Science Ltd. All rights reserved.
\end{abstract}

Keywords: A. Dislocations; Plasticity theory; Strain gradients; Material length scales; B. Higherorder solids; Metals; C. Computational plasticity

\footnotetext{
${ }^{*}$ Corresponding author. Tel.: +1-617-495-2848; fax: +1-617-495-9837.

E-mail address: hutchinson@husm.harvard.edu (J.W. Hutchinson).
} 


\section{Introduction}

The range of problems being studied within the framework of strain gradient plasticity theory is expanding steadily: flow and creep of polycrystals, two-phase alloys and reinforced composites (Smyshlyaev and Fleck, 1994, 1996; Gurtin, 2000), shear bands and other localizations (Aifantis, 1984; Zbib and Aifantis, 1989; de Borst and Muhlhaus, 1992; Mikkelsen, 1999; Sluys and Estrin, 2000), crack propagation (Wei and Hutchinson, 1997), microindentation (Begley and Hutchinson, 1998; Shu and Fleck, 1998; Nix and Gao, 1998), grain boundary effects in bi-crystals (Shu and Fleck, 1999; Shu et al., 2001). Most of the studies have employed one of three different types of phenomenological theories that have been put forward to incorporate the role the gradient of strain plays in increasing the effective flow strength of metals when plastic deformation takes place at the micron scale. Each of the three theories can be cast in a form which reduces to the classical $J_{2}$ plasticity theory (flow theory or deformation theory, depending on the version) in the limit when the length scales of the imposed deformation gradients are large compared to the material length parameters. Thus, the intention of all the continuum theories that have been proposed is to extend the validity of conventional plasticity down to roughly the micron scale where material length scale effects have been well documented. It is envisioned that the scale of the deformation phenomena is sufficiently large compared to dislocation spacing that a continuum description of plastic flow is justified. This would appear to be the case for most, if not all, of the problems cited above.

One type of theory, promulgated by Bassani and co-workers (Acharya and Bassani, 2000; Bassani et al., 2001) retains all the features of conventional $J_{2}$ flow theory but incorporates a dependence on plastic strain gradients into the incremental, or tangential, moduli. The great advantage of this class of theories is its ease of conversion into conventional numerical finite element codes. The theory has standard boundary conditions and introduces no higher-order stresses. It has been used to characterize rate-independent plastic deformation and is readily reformulated to accommodate creep, or other visco-plastic, behavior (Arsenlis and Parks, 1999; Busso et al., 2000). The main issue with this class of theories hinges on whether a standard theory is capable of embracing boundary and interface phenomena that may need to be modeled by non-standard boundary conditions. While the focus is not on this type of theory here, there will be examples that illustrate the necessity of additional boundary conditions in small-scale plasticity.

The second type of theory is that first proposed by Aifantis (1984) to ascribe a width to shear bands in metals, which are typically measured in microns. This theory has extra boundary conditions and possesses higher-order stress quantities, although the existence of the latter quantities has not been emphasized. The simplest version of the theory introduces one material length scale and employs as its measure of strain gradients the magnitude of the gradient of the amplitude of the plastic strain. This version is relatively straightforward to implement in a finite element code compared to nearly all other higher-order formulations, an advantage that cannot lightly be dismissed. A disadvantage is its inability, as formulated, to address a range of problems that appear to require more than just one material length parameter. The specifics of this requirement 
will be spelled out here. A generalization of this class of theories is given with two (or, possibly, three) material length parameters. The numerical implementation of the generalized version is not quite as straightforward as the simplest version, but it still retains significant advantages over most other higher-order formulations.

The third type of theory is the class of higher-order theories proposed and applied by Fleck and Hutchinson (1997). Although this type of theory will not be featured here, it is relevant to contrast its advantages and disadvantages with the class of theories under scrutiny, and this will be done at the end of the paper. It will be seen that the proposed new multiple parameter formulation gives quantitatively similar predictions in the plastic range to the corresponding theory of Fleck and Hutchinson. To motivate the present work, it can be remarked in advance that the class of theories being featured in this paper has several advantages over the original formulation proposed by Fleck and Hutchinson. One is the relative ease of numerical implementation, as already noted. The other relates to the manner in which the plastic strains are treated as variables in the theory, placing them on a footing similar to the displacements with implications for both the theoretical framework and numerical implementation.

The essential aspects of interest here can all be examined within the context of small strain/small rotation plasticity, and this restriction will be adopted. The spirit of the approach will be to develop a robust extension to $J_{2}$ theory to include the effects of strain gradients. We will attempt to identify a promising candidate theory with the aid of the available experimental data and current understanding of small-scale plasticity. Another objective is the extraction of the essential structure of this class of theories in a form not emphasized before.

\section{Measures of gradients of plastic strain rate}

Aifantis and coworkers employ the magnitude of the gradient of the conventional effective plastic strain rate, $\dot{\varepsilon}_{\mathrm{P}}=\sqrt{2 \dot{\varepsilon}_{i j}^{\mathrm{P}} \dot{\varepsilon}_{i j}^{\mathrm{P}} / 3}$, as the measure of the strain gradients. With $\dot{\varepsilon}_{\mathrm{P}, i} \dot{\varepsilon}_{\mathrm{P}, i} \equiv \vec{\nabla} \dot{\varepsilon}_{\mathrm{P}} \cdot \vec{\nabla} \dot{\varepsilon}_{\mathrm{P}}$ as the magnitude squared of this gradient, the present formulation of the Aifantis theory makes use of an effective plastic strain rate that includes gradient contributions and which is defined by

$$
\dot{E}_{\mathrm{P}}^{2}=\dot{\varepsilon}_{\mathrm{P}}^{2}+\ell_{*}^{2} \dot{\varepsilon}_{\mathrm{P}, i} \dot{\varepsilon}_{\mathrm{P}, i}
$$

This formulation has a single length parameter, $\ell_{*}$, which is required for dimensional consistency and whose role will be examined in detail.

It will be argued below that a one-parameter measure such as (1) does not have the scope to include the wide range of small-scale plasticity phenomena of interest. To generalize it, consistent with the objective of producing an extension of isotropic $J_{2}$ flow theory, we seek to identify invariants of the gradients of plastic strain rate, $\dot{\varepsilon}_{i j, k}^{\mathrm{P}}$, that are homogeneous of degree two. For this purpose, introduce the plastic strain gradient as

$$
\rho_{i j k}=\rho_{j i k}=\dot{\varepsilon}_{i j, k}^{\mathrm{P}}
$$


Incompressibility of plastic deformation implies $\rho_{i i k}=0$. As in Fleck and Hutchinson (1997), a unique orthogonal decomposition of the type given by Smyshlyaev and Fleck (1996) for third-order tensors is the starting point in identifying the invariants. The detailed steps of the decomposition follow those given by Smyshlyaev and Fleck for the analogous tensor $\eta_{i j k}=\eta_{j i k} \equiv u_{k, i j}$ defined in terms of the displacement field $u_{i}\left(x_{j}\right)$; there, incompressibility dictated $\eta_{i k k}=0$. The outcome is

$$
\rho_{i j k}=\rho_{i j k}^{(1)}+\rho_{i j k}^{(2)}+\rho_{i j k}^{(3)}
$$

with

$$
\begin{aligned}
& \rho_{i j k}^{(1)}=\rho_{i j k}^{\mathrm{S}}-\frac{1}{5}\left(\delta_{i j} \rho_{k p p}^{\mathrm{S}}+\delta_{i k} \rho_{j p p}^{\mathrm{S}}+\delta_{j k} \rho_{i p p}^{\mathrm{S}}\right), \\
& \rho_{i j k}^{(2)}=\frac{1}{3} e_{k i p} \chi_{p j}^{\mathrm{S}}+\frac{1}{3} e_{k j p} \chi_{p i}^{\mathrm{S}}, \\
& \rho_{i j k}^{(3)}=\frac{1}{3} e_{k i p} \chi_{p j}^{A}+\frac{1}{3} e_{k j p} \chi_{p i}^{A}+\frac{1}{5}\left(\delta_{i j} \rho_{k p p}^{\mathrm{S}}+\delta_{i k} \rho_{j p p}^{\mathrm{S}}+\delta_{j k} \rho_{i p p}^{\mathrm{S}}\right),
\end{aligned}
$$

where

$$
\begin{aligned}
& \rho_{i j k}^{\mathrm{S}}=\frac{1}{3}\left(\rho_{i j k}+\rho_{j k i}+\rho_{k i j}\right), \quad \chi_{i j}=e_{i q r} \rho_{j r q}, \\
& \chi_{i j}^{\mathrm{S}}=\frac{1}{2}\left(\chi_{i j}+\chi_{j i}\right) \quad \text { and } \quad \chi_{i j}^{A}=\frac{1}{2}\left(\chi_{i j}-\chi_{j i}\right) .
\end{aligned}
$$

Here, $\delta_{i j}$ is the Kronecker delta, $e_{i j k}$ is the permutation tensor, $\rho_{i j k}^{\mathrm{S}}$ is symmetric in all its indices, and $\chi_{i i}=0$ because $\rho_{i j k}=\rho_{j i k}$. Each of the three tensors on the right-hand side of (3) shares the same symmetry in the first two indices as $\rho_{i j k}$, and each has $\rho_{i i k}^{(m)}=0$. Moreover, they have been constructed such that they are mutually orthogonal in the sense that $\rho_{i j k}^{(m)} \rho_{i j k}^{(n)}=0$ for $m \neq n$. Since $\rho_{i j k}$ has only three independent invariants that are homogeneous of degree two, it follows that $\rho_{i j k}^{(m)} \rho_{i j k}^{(m)}$ for $m=1,3$ can be taken for that purpose. Useful identities for evaluating these invariants are

$$
\begin{aligned}
& \rho_{i j k}^{(1)} \rho_{i j k}^{(1)}=\rho_{i j k}^{\mathrm{S}} \rho_{i j k}^{\mathrm{S}}-\frac{4}{15} \rho_{k i i} \rho_{k j j}, \\
& \rho_{i j k}^{(2)} \rho_{i j k}^{(2)}=\frac{1}{3}\left(\chi_{i j} \chi_{i j}+\chi_{i j} \chi_{j i}\right), \\
& \rho_{i j k}^{(2)} \rho_{i j k}^{(2)}=\frac{3}{5}\left(\chi_{i j} \chi_{i j}-\chi_{i j} \chi_{j i}\right) .
\end{aligned}
$$

Contact can be made with the three invariants of the strain gradients introduced by Fleck and Hutchinson (1997) based on $\eta_{i j k}=\eta_{j i k} \equiv u_{k, i j}=\varepsilon_{k i, j}+\varepsilon_{k j, i}-\varepsilon_{i j, k}$. If one identifies the strains in this expression with the plastic strain rate components such that

$$
\eta_{i j k} \equiv \dot{\varepsilon}_{k i, j}^{\mathrm{P}}+\dot{\varepsilon}_{k j, i}^{\mathrm{P}}-\dot{\varepsilon}_{i j, k}^{\mathrm{P}}=\rho_{k i j}+\rho_{k j i}-\rho_{i j k},
$$

then $\eta_{k i i}$ vanishes; also, $\chi_{i j}$ as defined in (5) can be written as

$$
\chi_{i j}=\frac{1}{2} e_{i q r} \eta_{j q r} .
$$

The orthogonal decomposition, $\eta_{i j k}=\eta_{i j k}^{(1)}+\eta_{i j k}^{(2)}+\eta_{i j k}^{(3)}$, with $\eta_{k i i}^{(m)}=0$ for $m=1,3$ (cf. Fleck and Hutchinson, 1997) also provides three independent invariants of the plastic 
strain rate gradients: $\eta_{i j k}^{(m)} \eta_{i j k}^{(m)}, m=1,3$. The connections with the set in (6) are

$$
\eta_{i j k}^{(1)} \eta_{i j k}^{(1)}=\rho_{i j k}^{(1)} \rho_{i j k}^{(1)}, \quad \eta_{i j k}^{(2)} \eta_{i j k}^{(2)}=4 \rho_{i j k}^{(2)} \rho_{i j k}^{(2)}, \quad \eta_{i j k}^{(3)} \eta_{i j k}^{(3)}=\frac{8}{3} \rho_{i j k}^{(3)} \rho_{i j k}^{(3)} .
$$

For the multi-parameter theory, we take the measure of generalized effective plastic strain rate, $\dot{E}_{\mathrm{P}}$, to have precisely the same form as the effective strain in our earlier work:

$$
\begin{aligned}
\dot{E}_{\mathrm{P}}^{2} & =\dot{\varepsilon}_{\mathrm{P}}^{2}+\ell_{1}^{2} \eta_{i j k}^{(1)} \eta_{i j k}^{(1)}+\ell_{2}^{2} \eta_{i j k}^{(2)} \eta_{i j k}^{(2)}+\ell_{3}^{2} \eta_{i j k}^{(3)} \eta_{i j k}^{(3)} \\
& =\dot{\varepsilon}_{\mathrm{P}}^{2}+\ell_{1}^{2} \rho_{i j k}^{(1)} \rho_{i j k}^{(1)}+4 \ell_{2}^{2} \rho_{i j k}^{(2)} \rho_{i j k}^{(2)}+(8 / 3) \ell_{3}^{2} \rho_{i j k}^{(3)} \rho_{i j k}^{(3)},
\end{aligned}
$$

where again $\dot{\varepsilon}_{\mathrm{P}}^{2}=2 \dot{\varepsilon}_{i j}^{\mathrm{P}} \dot{\varepsilon}_{i j}^{\mathrm{P}} / 3$. The generalized effective plastic strain is intended as a phenomenological measure of the total dislocation density-dislocations that are statistically stored plus geometrically necessary dislocations induced by the strain gradients. The expression in (10) is the most general isotropic measure that is homogeneous of degree two in the plastic strain rates and their first gradients. It is positive definite, and the three length parameters, $\ell_{i}$, are required for dimensional consistency. As is the case for $\ell_{*}$ in (1), they set the scales at which the gradients become important. If the plastic strains are compatible, such that a displacement field exists with $\varepsilon_{i j}^{\mathrm{P}}=\left(u_{i, j}+u_{j, i}\right) / 2$, then the contributions depending on $\ell_{2}$ and $\ell_{3}$ vanish when the rotation rate gradients vanish (i.e. when $\chi_{i j}=0$, cf. (5)). The contribution depending on $\ell_{1}$ measures stretch rate gradients as well as rotation gradients. While this interpretation is no longer strictly true for general distributions of plastic strain, it, nevertheless, retains approximate validity and continues to be useful. Insight into the roles of the three gradient contributions will emerge from specific examples given later in the paper. An argument will be presented that the variety of plasticity phenomena at small scales dictates the necessity of more than one length parameter in the gradient description.

\section{Generalizations of classical $J_{2}$ theory}

As emphasized, the purpose here is to provide generalizations of the classical phenomenological isotropic plasticity theories, $J_{2}$ flow theory and $J_{2}$ deformation theory. It is surmised that these can be applied to a wide range of plasticity phenomena at the micron scale. The generalized theory incorporates a dependence on the gradients of plastic strain through the measure of the effective plastic strain, either (1) or (10), thereby bringing into play the new material length parameters. The formulations reduce to the respective conventional $J_{2}$ theories when either the material length parameters are set to zero or, equivalently, when the length scale characterizing the deformation field is large compared to the material length parameters. The flow theory is given first, followed by the deformation theory. The theory is restricted to small strains and rotations.

\subsection{The flow theory}

Although the statement of the theory given below differs in appearance from the form usually stated for the Aifantis theory, it is entirely equivalent. The elastic and 
plastic parts of the strains are denoted as $\varepsilon_{i j}^{\mathrm{e}}$ and $\varepsilon_{i j}^{\mathrm{P}}$ with the total strain, $\varepsilon_{i j}$, as their sum. The conventional effective plastic strain rate is $\dot{\varepsilon}_{\mathrm{P}}=\sqrt{2 \dot{\varepsilon}_{i j}^{\mathrm{P}} \dot{\varepsilon}_{i j}^{\mathrm{P}} / 3}$ with $\varepsilon_{\mathrm{P}}=\int \dot{\varepsilon}_{\mathrm{P}} \mathrm{d} t$. The present formulation explicitly recognizes the existence of higher-order stresses in the theory. Standard notation is used to denote the conventional stresses, $\sigma_{i j}=\sigma_{j i}$, their deviatoric stress components, $s_{i j}$, and the conventional effective stress, $\sigma_{\mathrm{e}}=\sqrt{3 s_{i j} s_{i j} / 2}$. The elastic properties of the material are taken to be isotropic with Young's modulus, $E$, Poisson's ratio, $v$, and with the moduli tensor, $C_{i j k l}$. As in the case of conventional $J_{2}$ flow theory, data in the form of the uniaxial tensile stress-strain curve are an input to the formulation. Specifically, the tangent hardening quantity, $h\left(\varepsilon_{\mathrm{P}}\right)=\mathrm{d} \sigma / \mathrm{d} \varepsilon_{\mathrm{P}}$, evaluated from uniaxial tensile data of stress $\sigma$ versus plastic strain $\varepsilon_{\mathrm{P}}$ will appear prominently.

The formulation under consideration is unusual in the sense that the free variables employed are $u_{i}$ and $\varepsilon_{\mathrm{P}}$, this being the feature that makes the formulation attractive for numerical work. The conventional plastic flow condition is assumed such that with the yield condition satisfied and $\dot{\varepsilon}_{\mathrm{P}} \geqslant 0$ (plastic loading),

$$
\dot{\varepsilon}_{i j}^{\mathrm{P}}=\dot{\varepsilon}_{\mathrm{P}} m_{i j}
$$

where $m_{i j}=(3 / 2) s_{i j} / \sigma_{\mathrm{e}}$; otherwise, $\dot{\varepsilon}_{i j}^{\mathrm{P}}=0$. Other details of the constitutive law will be given following the statements of the principle of virtual work and equilibrium.

Variations of $u_{i}, \varepsilon_{\mathrm{P}}$ and $\varepsilon_{\mathrm{P}, i}$ appear in the variational principles underlying the formulation, and a higher-order stress vector quantity $\tau_{i}$ arises naturally as the work conjugate to $\varepsilon_{\mathrm{P}, i}$. The internal virtual work increment, IVW, takes the form

$$
I V W=\int_{V}\left\{\sigma_{i j} \delta \varepsilon_{i j}^{\mathrm{e}}+Q \delta \varepsilon_{\mathrm{P}}+\tau_{i} \delta \varepsilon_{\mathrm{P}, i}\right\} \mathrm{d} V
$$

where $\sigma_{i j} \delta \varepsilon_{i j}^{\mathrm{e}}$ is the elastic work increment, $Q$ is defined as the work conjugate to the plastic strain $\varepsilon_{\mathrm{P}}$, and $\tau_{i} \delta \varepsilon_{\mathrm{P}, i}$ is the contribution due to the plastic strain gradients. Upon noting that $\sigma_{i j} \delta \varepsilon_{i j}^{\mathrm{P}}=\sigma_{\mathrm{e}} \delta \varepsilon_{\mathrm{P}}$ from (11), expression (12) is integrated by parts to give

$$
I V W=\int_{V}\left\{-\sigma_{i j, j} \delta u_{i}+\left(Q-\sigma_{\mathrm{e}}-\tau_{i, i}\right) \delta \varepsilon_{\mathrm{P}}\right\} \mathrm{d} V+\int_{S}\left\{\sigma_{i j} n_{j} \delta u_{i}+\tau_{i} n_{i} \delta \varepsilon_{\mathrm{P}}\right\} \mathrm{d} S,
$$

where $n_{i}$ is the unit outward normal to $S$. In the absence of body forces, the volume integral on the right-hand side of (13) vanishes, and we recover the usual equilibrium relation

$$
\sigma_{i j, j}=0
$$

in $V$, together with the relation for the generalized effective stress,

$$
Q=\sigma_{\mathrm{e}}+\tau_{i, i}
$$

The yield condition is specified by $Q=Q_{Y}$ where the evolution equation for the generalized yield stress $Q_{Y}$ emerges below. On combining (12)-(15), the principle 
of virtual work statement follows as

$$
\int_{V}\left\{\sigma_{i j} \delta \varepsilon_{i j}+\tau_{i, i} \delta \varepsilon_{\mathrm{P}}+\tau_{i} \delta \varepsilon_{\mathrm{P}, i}\right\} \mathrm{d} V=\int_{S}\left(T_{i} \delta u_{i}+t \delta \varepsilon_{\mathrm{P}}\right) \mathrm{d} S,
$$

where the traction quantities $T_{i}$ and $t$ on the boundary $S$ satisfy the relations

$$
T_{i}=\sigma_{i j} n_{j} \quad \text { and } \quad t=\tau_{i} n_{i} \text { on } S .
$$

Note that the stress is symmetric $\left(\sigma_{i j}=\sigma_{j i}\right)$ and the conventional relation between the stress tensor and the surface traction vector is preserved. The contributions in (16) from $Q, t$ and $\tau_{i}$ are absent where the yield condition is not satisfied. That higher-order stresses are intrinsic to the theory is evident from the virtual work statement (16). In particular, the rate of work across any exterior or internal surface, $S$, is $\sigma_{i j} n_{j} \dot{u}_{i}+\tau_{i} n_{i} \dot{\dot{\varepsilon}} \mathrm{P}$ and not $\sigma_{i j} n_{j} \dot{u}_{i}$. It does not seem to be widely appreciated that an equilibrium state of the body in this class of theories cannot be interpreted in terms of conventional stresses alone.

The principle of virtual work for incremental problems reads

$$
\begin{aligned}
& \int_{V}\left\{\dot{\sigma}_{i j} \delta \dot{\varepsilon}_{i j}^{\mathrm{e}}+\dot{Q} \delta \dot{\varepsilon}_{\mathrm{P}}+\dot{\tau}_{i} \delta \dot{\varepsilon}_{\mathrm{P}, i}\right\} \mathrm{d} V=\int_{V}\left\{\dot{\sigma}_{i j} \delta \dot{\varepsilon}_{i j}+\dot{\tau}_{i, i} \delta \dot{\varepsilon}_{\mathrm{P}}+\dot{\tau}_{i} \delta \dot{\varepsilon}_{\mathrm{P}, i}\right\} \mathrm{d} V \\
& =\int_{S}\left(\dot{T}_{i} \delta \dot{u}_{i}+\dot{t} \delta \dot{\varepsilon}_{\mathrm{P}}\right) \mathrm{d} S .
\end{aligned}
$$

The equations for equilibrium and surface tractions are the rate forms of (14) and (17), respectively.

While the effective strain rate in (1) is already in a form explicitly displaying $\dot{\varepsilon}_{\mathrm{P}}$ and $\dot{\varepsilon}_{\mathrm{P}, i}$, it must be rendered in such a form for the generalized rate in (10). From (11),

$$
\rho_{i j k}=\dot{\varepsilon}_{i j, k}^{\mathrm{P}}=\dot{\varepsilon}_{\mathrm{P}, k} m_{i j}+\dot{\varepsilon}_{\mathrm{P}} m_{i j, k}
$$

and after performing the orthogonal decomposition of $\rho_{i j k}$, expression (10) for the effective strain rate can be recast as

$$
\dot{E}_{\mathrm{P}}^{2}=\dot{\varepsilon}_{\mathrm{P}}^{2}+A_{i j} \dot{\varepsilon}_{\mathrm{P}, i} \dot{\varepsilon}_{\mathrm{P}, j}+B_{i} \dot{\varepsilon}_{\mathrm{P}, i} \dot{\varepsilon}_{\mathrm{P}}+C \dot{\varepsilon}_{\mathrm{P}}^{2}
$$

In general, the explicit expressions for $A_{i j}\left(=A_{j i}\right), B_{i}$ and $C$ depend on the three material length parameters, as well as on position when $m_{i j}$ depends on position. General expressions for these coefficients are given in the Appendix, and illustrations will be given later for specific problems. Note that (1) is formally included in (20) if $A_{i j}=\ell_{*}^{2} \delta_{i j}, B_{i}=0$ and $C=0$, even though (1) is not a special case of (10).

The incremental boundary value problem can be stated as a minimum principle $\delta I=0$, with

$$
\begin{aligned}
I\left(\dot{u}_{i}, \dot{\varepsilon}_{\mathrm{P}}\right)= & \frac{1}{2} \int_{V}\left\{C_{i j k l}\left(\dot{\varepsilon}_{i j}-\dot{\varepsilon}_{\mathrm{P}} m_{i j}\right)\left(\dot{\varepsilon}_{k l}-\dot{\varepsilon}_{\mathrm{P}} m_{k l}\right)+h\left(E_{\mathrm{P}}\right) \dot{E}_{\mathrm{P}}^{2}\right\} \mathrm{d} V \\
& -\int_{S_{T}}\left(\dot{T}_{i}^{0} \dot{u}_{i}+\dot{t}^{0} \dot{\varepsilon}_{\mathrm{P}}\right) \mathrm{d} S .
\end{aligned}
$$

Here, $\dot{T}_{i}^{0}$ and $\dot{t}^{0}$ are prescribed traction rates on $S_{T}$, and $E_{\mathrm{P}}=\int \dot{E}_{\mathrm{P}} \mathrm{d} t$ is the accumulated effective plastic strain. A proof of this minimum principle, together with a discussion 
of the uniqueness are summarised in the Appendix. The first term in the volume integral is the incremental elastic work rate density while the second represents the plastic work rate density. Apart from the explicit choice of $h\left(E_{\mathrm{P}}\right)$ in $(21)$ and the inclusion of the higher-order traction rate, $\dot{t}^{0}$, on the boundary, principle (21) is precisely that introduced by Muhlhaus and Aifantis (1991) in their presentation of a variational principle governing the one-parameter theory based on (1). Subsequently, de Borst and Muhlhaus (1992) laid out a numerical finite element scheme for the one-parameter theory based on (21). When the material length parameters are zero, this principle and the associated equations reduce to the classical $J_{2}$ flow theory, although unconventionally due to the special role of $\varepsilon_{\mathrm{P}}$.

As indicated, we propose to evaluate the uniaxial hardening function $h=\mathrm{d} \sigma / \mathrm{d} \varepsilon_{\mathrm{P}}$ in $(21)$ at the generalized effective plastic strain, $E_{\mathrm{P}}$, rather than at $\varepsilon_{\mathrm{P}}$. This is the one aspect where the present formulation differs from versions of the one-parameter Aifantis theory that have been used by other investigators. Some formulations take $h\left(\varepsilon_{\mathrm{P}}\right)$ in $(21)$, and others separate $h \dot{\varepsilon}_{\mathrm{P}}^{2}$ from the gradient term in a different manner such that the coefficient of the gradient term is independent of position. While the specific choice is important for its quantitative effect on predictions, it is not critical to the structure of the formulation. One argument for the choice in (21) rests on the idea that it is the total dislocation density that primarily influences hardness evolution, and $E_{\mathrm{P}}$ is intended to measure this density. A more compelling reason for this choice will emerge shortly when the deformation theory version of the theory is presented.

The field equations in the loading regions produced from the variational equation $\delta I=0$ are the rate form of the equilibrium equations (14) and associated boundary conditions (17). In addition, by identifying $\dot{Q} \equiv \dot{\sigma}_{\mathrm{e}}+\dot{\tau}_{i, i}$ and $\dot{\tau}_{i}$ in (18) with the requisite terms in the resulting field equations, one obtains the remaining constitutive equations governing plastic loading $\left(\dot{Q}=\dot{Q}_{Y}\right)$ as

$$
\begin{aligned}
& \dot{Q}_{Y}=h\left(E_{\mathrm{P}}\right)\left(\dot{\varepsilon}_{\mathrm{P}}+\frac{1}{2} B_{i} \dot{\varepsilon}_{\mathrm{P}, i}+C \dot{\varepsilon}_{\mathrm{P}}\right), \\
& \dot{\tau}_{i}=h\left(E_{\mathrm{P}}\right)\left(A_{i j} \dot{\varepsilon}_{\mathrm{P}, j}+\frac{1}{2} B_{i} \dot{\varepsilon}_{\mathrm{P}}\right) .
\end{aligned}
$$

For the one-parameter theory based on (1), the variational principle (21) gives

$$
\begin{aligned}
& \dot{Q}_{Y}=h\left(E_{\mathrm{P}}\right) \dot{\varepsilon}_{\mathrm{P}}, \\
& \dot{\tau}_{i}=\ell_{*}^{2} h\left(E_{\mathrm{P}}\right) \dot{\varepsilon}_{\mathrm{P}, i}
\end{aligned}
$$

and the effective stress rate follows from (15), (24) and (25) as

$$
\dot{\sigma}_{\mathrm{e}}=\dot{Q}-\dot{\tau}_{i, i}=h \dot{\varepsilon}_{\mathrm{P}}-\ell_{*}^{2}\left(h \dot{\varepsilon}_{\mathrm{P}, i}\right)_{, i}
$$

If there is no spatial variation of $\ell_{*}^{2} h$ in the second term (as is the case in some alternative formulations), this equation becomes

$$
\dot{\sigma}_{\mathrm{e}}=\dot{Q}-\dot{\tau}_{i, i}=h \dot{\varepsilon}_{\mathrm{P}}-\ell_{*}^{2} h \nabla^{2} \dot{\varepsilon}_{\mathrm{P}}
$$


In Aifantis's (1984) original formulation, a "hardening" equation of this general form involving the Laplacian of the effective plastic strain rate was proposed as the starting point of the new theory. The presence of the higher-order stress quantities in the theory makes it difficult to interpret this equation in a conventional manner, and this may be the source of some of the confusion which has surrounded it. ${ }^{1}$ In particular, the current value of $\sigma_{\mathrm{e}}$ is not a meaningful measure of the current state of hardness. An alternative is mentioned in the next subsection.

The specification of the generalized $J_{2}$ flow theory is complete. Illustrations of the theory and discussions of particular choices of boundary conditions will be given in connection with solutions for the basic problems presented in the next section.

\subsection{The deformation theory}

We end this section with the presentation of a total (versus incremental) formulation that coincides exactly with the flow theory stated above when proportional stressing occurs throughout the body. Although proportional stressing is seldom satisfied precisely, it will be demonstrated that the generalized deformation theory can nevertheless provide very accurate approximations to flow theory solutions. The formulation has an unusual form for a deformation theory in that it employs the total conventional effective "plastic" strain, $\varepsilon_{\mathrm{P}}$, as a variable. However, as in the case of the flow theory, the generalized deformation theory reduces to classical $J_{2}$ deformation theory when the material length parameters are set to zero. The applicability and utility of deformation theory is well appreciated (Budiansky, 1959). Not only does it permit the closed-form solution to certain basic problems, it also enables theoretical developments that would not otherwise exist, e.g. the $J$-integral in crack mechanics. For numerical work, a deformation theory has the notable advantage that solutions at arbitrary loads can be obtained directly by iteration without recourse to solving a sequence of incremental problems at smaller loads.

To define the deformation theory, let $\varepsilon_{i j}^{\mathrm{P}}=\varepsilon_{\mathrm{P}} m_{i j}\left(\varepsilon_{\mathrm{P}} \geqslant 0\right)$, where, again, $m_{i j}=(3 / 2) s_{i j} / \sigma_{\mathrm{e}}$. Next, form the generalized total effective strain using the definition in (10), but now in terms of the total "plastic" strains and their gradients, $\rho_{i j k} \equiv \varepsilon_{i j, k}^{\mathrm{P}}=\varepsilon_{\mathrm{P}, k} m_{i j}+\varepsilon_{\mathrm{P}} m_{i j, k}$, with the result

$$
E_{\mathrm{P}}^{2}=\varepsilon_{\mathrm{P}}^{2}+A_{i j} \varepsilon_{\mathrm{P}, i} \varepsilon_{\mathrm{P}, j}+B_{i} \varepsilon_{\mathrm{P}, i} \varepsilon_{\mathrm{P}}+C \varepsilon_{\mathrm{P}}^{2}
$$

The factors $A_{i j}, B_{i}$ and $C$ are identical to those in the flow theory expression (20) when the distribution of $m_{i j}$ is the same (see the Appendix). Define a potential energy

\footnotetext{
${ }^{1}$ It has been suggested that a "hardening" contribution proportional to $\sqrt{\dot{\varepsilon}_{\mathrm{P}, i} \dot{\varepsilon}_{\mathrm{P}, i}}$ can be included in (27) in place of the term containing $\nabla^{2} \dot{\dot{\varepsilon}}$. We believe that proposal is not physically sound. A term of this type could not arise from a variational principle such as (21). More fundamentally, a contribution of this form replacing $\nabla^{2} \dot{\varepsilon}_{\mathrm{P}}$ would turn (27) into a first-order partial differential equation which is inherently inconsistent with boundary condition requirements for solid bodies: the field equations would state an initial value problem with one-sided boundary conditions, rather than a boundary value problem. This can be appreciated by noting that one-dimensional problems require boundary conditions to be applied at both ends of the interval.
} 
functional as

$$
\begin{aligned}
\Phi\left(u_{i}, \varepsilon_{\mathrm{P}}\right)= & \int_{v}\left\{\frac{1}{2} C_{i j k l}\left(\varepsilon_{i j}-\varepsilon_{\mathrm{P}} m_{i j}\right)\left(\varepsilon_{k l}-\varepsilon_{\mathrm{P}} m_{k l}\right)+\int_{0}^{E_{\mathrm{P}}} \sigma\left(\tilde{\varepsilon}_{\mathrm{P}}\right) \mathrm{d} \tilde{\varepsilon}_{\mathrm{P}}\right\} \mathrm{d} V \\
& -\int_{S_{T}}\left(T_{i}^{0} u_{i}+t^{0} \varepsilon_{\mathrm{P}}\right) \mathrm{d} S .
\end{aligned}
$$

Then, the actual solution minimises $\Phi\left(u_{i}, \varepsilon_{\mathrm{P}}\right)$ provided $C_{i j k l}$ is positive definite and $h$ is positive. As before, $\sigma\left(\varepsilon_{\mathrm{P}}\right)$ denotes data from the uniaxial tensile stress-strain curve of the material. The first contribution to the volume integral is the elastic energy density while the second is the plastic work density. In classical deformation theory the plastic work density is $\int_{0}^{\varepsilon_{\mathrm{P}}} \sigma\left(\tilde{\varepsilon}_{\mathrm{P}}\right) \mathrm{d} \tilde{\varepsilon}_{\mathrm{P}}$. Here, the plastic work density is evaluated at $E_{\mathrm{P}}$ rather than $\varepsilon_{\mathrm{P}}$.

The field equations, (14) and (15), and boundary conditions (17) associated with $\delta \Phi=0$ for all admissible $\delta u_{i}$ and $\delta \varepsilon_{\mathrm{P}}$ are obtained in a straightforward manner. In addition, one obtains the constitutive relations,

$$
\begin{aligned}
Q & =\frac{\sigma\left(E_{\mathrm{P}}\right)}{E_{\mathrm{P}}}\left(\varepsilon_{\mathrm{P}}+\frac{1}{2} B_{i} \varepsilon_{\mathrm{P}, i}+C \varepsilon_{\mathrm{P}}\right), \\
\tau_{i} & =\frac{\sigma\left(E_{\mathrm{P}}\right)}{E_{\mathrm{P}}}\left(A_{i j} \varepsilon_{\mathrm{P}, j}+\frac{1}{2} B_{i} \varepsilon_{\mathrm{P}}\right) .
\end{aligned}
$$

Consider boundary valued problems specified by a single load parameter $\lambda$ which is increased monotonically. Using an approach similar to that used for the classical theories, one can show that the solution to the boundary value problem for the deformation theory coincides with the solution for the corresponding flow theory when the deformation theory solution obeys proportional stressing. The details of this proof are omitted. Proportional stressing in the present context requires of the deformation theory solution that the stress components (both $\sigma_{i j}$ and $\tau_{i}$ ) increase monotonically at each point in fixed proportion. One special class of problems satisfying proportional stressing are solids modeled by a pure power uniaxial stress-strain curve, i.e. which neglect elasticity and have $\sigma \propto \varepsilon_{\mathrm{P}}^{N}$. Pure power law materials are often used to model behavior when plastic strains are large compared to elastic strains. Solutions to pure power law problems can be obtained with the present formulation as the limit computed for increasingly large Young's modulus. The shear problem discussed first in the next section exhibits proportional stressing even though it includes both elastic and plastic strains. For all but a very few special problems, however, the presence of elasticity prevents the precise satisfaction of proportional stressing. Nevertheless, solution of the wire torsion and void growth problems in the next section using both flow and deformation theory indicates that the deformation theory provides a highly accurate approximation to the flow theory solution even when the conditions for proportional stressing are not met precisely.

The presence of $h\left(E_{\mathrm{P}}\right)$ in the variational functional (21) for the flow theory is directly linked to the choice of the generalized plastic work density, $\int_{0}^{E_{\mathrm{P}}} \sigma\left(\tilde{\varepsilon}_{\mathrm{P}}\right) \mathrm{d} \tilde{\varepsilon}_{\mathrm{P}}$, in (29). If any other choice for $h$ had been made, the coincidence of solutions for the two 
types of theories under proportional loading would not occur. Recall that $h\left(E_{\mathrm{P}}\right)$ is $\mathrm{d} \sigma\left(\varepsilon_{\mathrm{P}}\right) / \mathrm{d} \varepsilon_{\mathrm{P}}$ evaluated at $E_{\mathrm{P}}$. The incremental functional (21) arises out of a second-order expansion of (29) about the current state. The generalized plastic strain $E_{\mathrm{P}}$ has been introduced as a phenomenological measure of dislocation density resulting from plastic strains (statistically stored dislocations) and their gradients (geometrically necessary dislocations). The generalized plastic work density provides perhaps the clearest and most elementary insight to the role played by $E_{\mathrm{P}}$ in the present theories. Simply stated, the theory postulates that the plastic work expended in bringing a material element subject to both plastic strains and plastic strain gradients to a generalized plastic strain $E_{\mathrm{P}}$ is the same as occurs in deforming that element to the plastic strain $E_{\mathrm{P}}$ in uniaxial tension. By the same token, the plastic flow resistance at the current state is reflected by $\sigma\left(E_{\mathrm{P}}\right)$. In effect, the formulation assumes that geometrically necessary dislocations and statistically stored dislocations increase the flow resistance in the same way.

It is also worth emphasizing that the present formulations tie hardening to the gradients of plastic strain $\varepsilon_{\mathrm{P}, i}$ and not to $\nabla^{2} \varepsilon_{\mathrm{P}}$, consistent with basic notions of the role of geometrically necessary dislocations. Instead, $\nabla^{2} \varepsilon_{\mathrm{P}}$ emerges in the resulting field equations as a byproduct of the more fundamental role of the plastic strain gradients. As mentioned already, the interpretation of hardening from an equation such as (27) is obscured by the presence of higher-order stresses that alter the conventional role of $\sigma_{\mathrm{e}}$.

\section{Solutions to problems and their implications}

Three problems are solved and interpreted in this section: (1) shearing of a layer sandwiched between two substrates, (2) wire torsion, and (3) expansion of a spherical void. This spectrum of problems permits one to draw conclusions about the material length parameters introduced in Section 2. In each study, a Ramberg-Osgood curve is taken to characterize the uniaxial tensile stress-strain curve of the solid. Specifically,

$$
\varepsilon / \varepsilon_{0}=\sigma / \sigma_{0}+\left(\sigma / \sigma_{0}\right)^{n}
$$

where $\varepsilon_{0}=\sigma_{0} / E$ and $E$ is Young's modulus. For this choice, $h\left(E_{\mathrm{P}}\right)=N E\left(E_{\mathrm{P}} / \varepsilon_{0}\right)^{N-1}$ with $N=1 / n$. Flow theory has been employed to generate the solutions in all three cases, however the wire torsion and void growth problems were also solved using deformation theory. As already noted, the respective flow and deformation theory solutions coincide for the shear problem.

\subsection{Shearing of a layer sandwiched between two substrates}

Consider an infinite elastic plastic layer, $-\infty<x_{1}<\infty$, of height $2 L,-L<x_{2}<L$, where each face is bonded to a rigid substrate. The substrates are displaced such that top and bottom of the layer undergo relative shearing displacements, $u_{1}=U$ and $u_{1}=-U$ at $x_{2}= \pm L$, respectively. The only non-zero displacement is $u_{1}\left(x_{2}\right)$, which is assumed to be independent of $x_{1}$. The one non-zero strain quantity is the shearing strain $\gamma\left(x_{2}\right)=u_{1}^{\prime}$ 
with ()$^{\prime} \equiv \mathrm{d}() / \mathrm{d} x_{2}$. With $\gamma_{\mathrm{P}}\left(x_{2}\right)$ denoting the plastic shear strain, the effective plastic strain rate defined in (10) is found to be

$$
\dot{E}_{\mathrm{P}}^{2}=\frac{1}{3} \dot{\gamma}_{\mathrm{P}}^{2}+\frac{1}{3}\left(\frac{4}{15} \ell_{1}^{2}+\frac{1}{3} \ell_{2}^{2}+\frac{2}{5} \ell_{3}^{2}\right) \dot{\gamma}_{\mathrm{P}}^{\prime 2}
$$

The corresponding expression for the single length scale version of theory (1) is

$$
\dot{E}_{\mathrm{P}}^{2}=\frac{1}{3} \dot{\gamma}_{\mathrm{P}}^{2}+\frac{1}{3} \ell_{*}^{2} \dot{\gamma}_{\mathrm{P}}^{\prime 2}
$$

Variational functional (21) for both formulations reduces to

$$
I\left(\dot{u}_{\mathrm{i}}, \dot{\gamma}_{\mathrm{P}}\right)=\frac{1}{2} \int_{-L}^{L}\left\{G\left(\dot{\gamma}-\dot{\gamma}_{\mathrm{P}}\right)^{2}+\frac{h\left(E_{\mathrm{P}}\right)}{3}\left(\dot{\gamma}_{\mathrm{P}}^{2}+\ell^{2} \dot{\gamma}_{\mathrm{P}}^{\prime 2}\right)\right\} \mathrm{d} x_{2}
$$

with $G$ as the elastic shear modulus, $\dot{\gamma}=\dot{u}_{1}^{\prime}$, and where $\ell$ depends on the formulation according to

$$
\ell \equiv \sqrt{\frac{4}{5} \ell_{1}^{2}+\ell_{2}^{2}+\frac{6}{5} \ell_{3}^{2}} \text { for }(10) \text { and } \ell \equiv \ell_{*} \quad \text { for }(1)
$$

Boundary conditions on the displacement rate at the top and bottom of the layer require $\dot{u}_{1}= \pm \dot{U}$, respectively. If these were the only conditions imposed at the boundaries with the substrates, the solution would be a uniform shearing of the layer with no gradients. This can be seen immediately by noting that the solution to this problem based on the classical $J_{2}$ theory has no strain gradients. It obviously satisfies all the field equations. (Uniqueness of solution can be proved directly from either (21) or (35), see Appendix A.) Strain gradients come into play in the shear problem only if the boundaries are assumed to constrain the plastic flow. If the substrates are rigid, or elastic but stiff, dislocations in the layer will be blocked as they approach the boundaries and arrest at a standoff distance; Shu et al. (2001) have recently simulated such behavior in a discrete dislocation calculation of constrained plastic flow within a crystalline layer. A continuum model of this situation must require the plastic strain to vanish at such a boundary, i.e. $\gamma_{\mathrm{P}}=0$ at $x_{2}= \pm L$. By contrast, dislocations approaching a free surface are free to pass out leaving steps and producing unconstrained plastic strain at the surface.

The field equation generated by the requirement that $\delta I=0$ for all admissible $\delta \dot{u}_{1}$ gives $G\left(\dot{\gamma}-\dot{\gamma}_{\mathrm{P}}\right)=\dot{S}$ where $\dot{S}$ is the shear force per unit area acting at the boundaries $\left(\dot{\gamma}_{\mathrm{P}}=0\right.$ at the boundaries implies that only $\sigma_{12}$ does work at the boundaries). Next, variations with respect to admissible $\delta \dot{\gamma}_{\mathrm{P}}$ give

$$
-\ell^{2}\left(h\left(E_{\mathrm{P}}\right) \dot{\gamma}_{\mathrm{P}}^{\prime}\right)^{\prime}+h\left(E_{\mathrm{P}}\right) \dot{\gamma}_{\mathrm{P}}=3 \dot{S} \quad \text { with } \quad \dot{\gamma}_{\mathrm{P}}=0 \text { at } x_{2}= \pm L .
$$

The solution for an incremental step of shear can be obtained by a numerical analysis of the ordinary differential equation, or it can be obtained by a finite element discretization of the functional in (35) and subsequent minimization with respect to the nodal amplitudes. The results presented in Figs. 1 and 2 have been obtained by the second method using linear elements for $\dot{u}_{1}$ and $\dot{\gamma}_{\mathrm{p}}$. Although this problem is one dimensional, this simple approach generalizes to two and three dimensions since linear elements preserve continuity of displacements and plastic strains, yielding total strains and plastic strain gradients that are uniform within each element. The plastic shear 


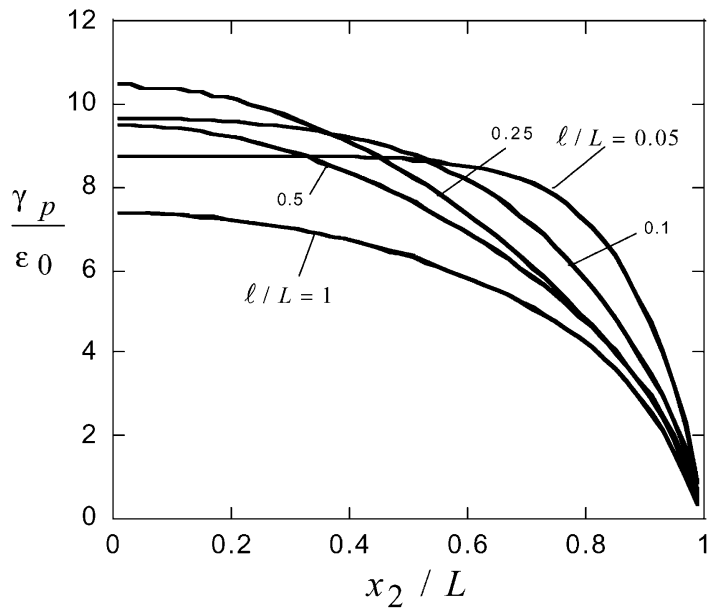

Fig. 1. Distribution of plastic shear strain across the upper half of the sheared layer at $U /\left(\varepsilon_{0} L\right)=10$ for various values of $\ell / L$ where $\ell$ is defined for each of the formulations in (36). The tensile stress-stress strain curve of the material is given by (32) with $N=0.2$ and $v=0.3$. The calculation employs 50 elements across the half-width of the layer and reaches $U /\left(\varepsilon_{0} L\right)=10$ in 100 incremental steps.

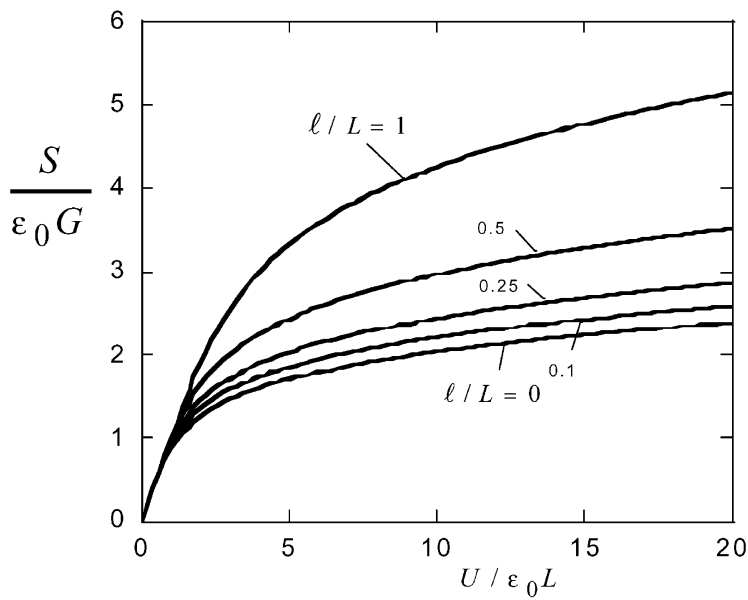

Fig. 2. Effect of the material length parameter $\ell$ on the overall relation between the shear traction and the shearing displacement for the elastic-plastic layer $(N=0.2$ and $v=0.3)$.

strain distribution in Fig. 1 displays a boundary layer which occupies a diminishing fraction of the layer thickness decreases as $\ell / L$ decreases. When $\ell / L$ is increased from zero to unity, the overall shear traction $S$ is elevated by more than a factor of two, see Fig. 2. Noticeable departures from the classical limit are seen for the material length scale as small as $\ell / L=0.1$. By (36), results of both the multiple and single-parameter models are covered by the plots in Figs. 1 and 2 if $\ell$ is identified with the appropriate length parameters. The fact that the deformation theory and flow theory predictions 
coincide for this problem follows directly from the homogeneity of the left-hand side of the ordinary differential equation (37).

\subsection{Wire torsion}

A solid cylindrical wire of radius $R$ is considered. A standard cylindrical coordinate system $(r, \theta, z)$ is employed. When the material is isotropic and the wire is twisted monotonically, the total shear strain is a known function of position according to $\gamma=\alpha r$ where $\alpha$ is the twist per unit length. The single nonzero conventional stress component is $\sigma_{\theta_{L}}$, and the plastic component of the shear strain is an unknown function of $r, \gamma_{\mathrm{P}}(r)$. By (11), the plastic strain rate is in the direction of the shear stress such that

$$
\dot{\boldsymbol{\varepsilon}}^{\mathrm{P}}=\frac{1}{2} \dot{\gamma}_{\mathrm{P}}\left(\vec{e}_{\theta} \vec{e}_{z}+\vec{e}_{z} \vec{e}_{\theta}\right) \text {. }
$$

The amplitude and the direction of the plastic strain rate are both functions of position. The nonzero components of the gradients of plastic strain rate defined in (2) are

$$
\rho_{\theta z r}=\rho_{z \theta r}=\frac{1}{2} \dot{\gamma}_{\mathrm{P}}^{\prime}, \quad \rho_{r z \theta}=\rho_{z r \theta}=-\frac{1}{2} r^{-1} \dot{\gamma}_{\mathrm{P}}
$$

The conventional effective plastic strain rate is given by $\dot{\varepsilon}_{\mathrm{P}}^{2}=\dot{\gamma}_{\mathrm{P}}^{2} / 3$ and the generalized effective plastic strain rate as defined in (10) is found to be

$$
\dot{E}_{\mathrm{P}}^{2}=\frac{1}{3} \dot{\gamma}_{\mathrm{P}}^{2}+\frac{1}{6} \ell_{1}^{2}\left(\dot{\gamma}_{\mathrm{P}}^{\prime}-r^{-1} \dot{\gamma}_{\mathrm{P}}\right)^{2}+\frac{4}{3} \ell_{2}^{2}\left(\dot{\gamma}_{\mathrm{P}}^{\prime 2}+r^{-1} \dot{\gamma}_{\mathrm{P}}^{\prime} \dot{\gamma}_{\mathrm{P}}+r^{-2} \dot{\gamma}_{\mathrm{P}}^{2}\right)
$$

The parameter $\ell_{3}$ does not enter the problem.

For the Aifantis-type formulation, $\dot{\varepsilon}_{\mathrm{P}, i} \dot{\varepsilon}_{\mathrm{P}, i}=\dot{\gamma}_{\mathrm{P}}^{\prime 2} / 3$ and the effective rate in (1) is

$$
\dot{E}_{\mathrm{P}}^{2}=\frac{1}{3} \dot{\gamma}_{\mathrm{P}}^{2}+\frac{1}{3} \ell_{*}^{2} \dot{\gamma}_{\mathrm{P}}^{\prime 2} .
$$

This measure depends only on the gradient of the plastic strain rate amplitude. Wire torsion illustrates the point made earlier that $\dot{\varepsilon}_{\mathrm{P}, i} \dot{\varepsilon}_{\mathrm{P}, i}$ does not provide a positive-definite measure of the full gradient of the plastic strain rate when the direction of the plastic strain rates is a function of position. For example, if at any point $\dot{\gamma}_{\mathrm{P}}^{\prime}$ vanishes, then the effective rate in (41) has no gradient contribution whereas (40) does. This will be seen to have direct bearing on the wire torsion problem.

The functional (21) governing the problem for the distribution of the plastic strain rate for each of the two formulations is

$$
I\left(\dot{\gamma}_{\mathrm{P}}\right)=\frac{1}{2} \int_{0}^{R}\left\{G\left(\dot{\alpha} r-\dot{\gamma}_{\mathrm{P}}\right)^{2}+h\left(E_{\mathrm{P}}\right) \dot{E}_{\mathrm{P}}^{2}\right\} 2 \pi r \mathrm{~d} r .
$$

If it is assumed that there is no constraint of the plastic flow at the surface of the wire, ${ }^{2}$ then the natural boundary condition associated with the variational principle is $\dot{\tau}_{i} n_{i}=0$. For the one parameter version, this requires $\dot{\gamma}_{\mathrm{P}}^{\prime}=0$ at $r=R$, while the condition from (23) for the multiple parameter involves both $\dot{\gamma}_{\mathrm{P}}$ and its derivative. Solutions to these problems have also been generated by a one-dimensional finite element analysis. In

\footnotetext{
${ }^{2}$ If the surface of the wire were covered by a very thin elastic coating that blocked dislocations, analogous to passivation layers laid down on thin films, a more appropriate boundary condition would be $\dot{\gamma}_{\mathrm{P}}=0$. A larger torque would then be required to attain a given twist. This example illustrates the scope of a higher-order theory to address nonstandard boundary and interface conditions.
} 


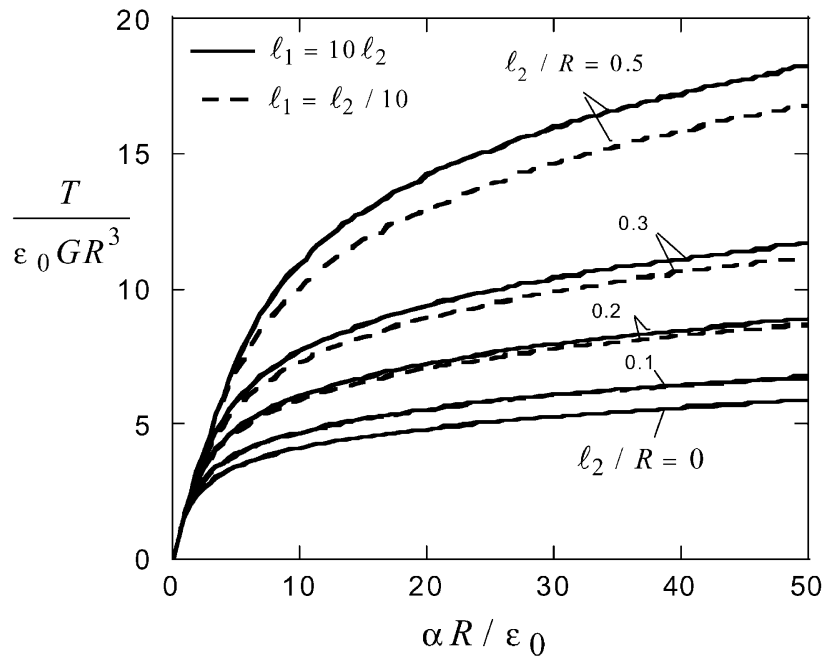

Fig. 3. Torque versus twist based on the general formulation for a solid cylindrical wire of radius $R$ with $N=0.2$ and $v=0.3$. The length parameter $\ell_{2}$ is the most important in determining the size effect in wire torsion; $\ell_{1}$ has very little influence, and $\ell_{3}$ does not enter the problem. The calculation employs 100 elements from the center of the wire to its free surface and it reaches $\alpha R / \varepsilon_{0}=50$ in 500 incremental steps. (Elastic compressibility of the wire does not play a role in the solution, however the value of Poisson's ratio enters into the normalization used when evaluating $G / E$.)

this case, only representations for $\dot{\gamma}_{\mathrm{P}}$ and its first derivative are required. Results in the form of torque as a function of twist are given in Fig. 3 for the theory based on (10). It is seen immediately that the first material length parameter, $\ell_{1}$, is unimportant in wire torsion: varying $\ell_{1}$ from a factor of $10 \ell_{2}$ to $\ell_{2} / 10$ has little effect upon the solution. The corresponding plots for the version of the theory based on (1) are given in Fig. 4. The responses based on the general formulation display an immediate increase in the torque due to gradient effects as soon as the wire begins to deform plastically, while torque increases predicted from the single length parameter formulation using (41) develop much more gradually. The absence of a gradient effect for the single-parameter case in the early stages of plastic flow lies in the fact that enforcement of the natural boundary condition $\dot{\gamma}_{\mathrm{P}}^{\prime}=0$ at $r=R$ causes the gradient solution to co-incide with the classical solution in the early stages of yield. Thus, the measure based on (41), which only involves $\dot{\gamma}_{\mathrm{p}}^{\prime}$, requires the plastic deformation to extend well into the interior of the wire before gradient effects become significant. In this connection, it should be mentioned that the predictions for wire torsion based on the one-parameter version given by Aifantis (1999) have some inaccuracy because the natural boundary condition $\left(\dot{\gamma}_{\mathrm{P}}^{\prime}=0\right.$ at $\left.r=R\right)$ has not been enforced.

Insight into why $\ell_{2}$ is the controlling parameter in wire torsion can be acquired by considering the classical solution in the absence of gradient effects. The classical solution in the limit in which the elastic strains are small compared to the plastic strains has $\dot{\gamma}_{\mathrm{P}}=\dot{\alpha} r$. For this distribution, the gradient contribution to $\dot{E}_{\mathrm{P}}^{2}$ in $(40)$ is $4 \ell_{2}^{2} \dot{\alpha}^{2}$. 


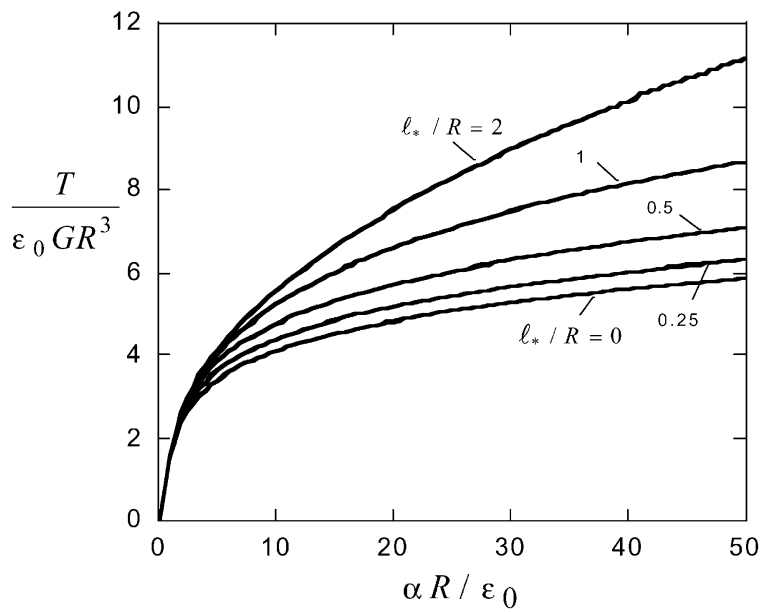

Fig. 4. Torque versus twist based on the one-parameter formulation for a solid wire of radius $R$ with $N=0.2$ and $v=0.3$. The calculation details are similar to those stated in Fig. 3 .

The "stretching" contribution involving $\ell_{1}$ vanishes. This limiting deformation can be regarded as one involving only rotation gradients. When strain gradient effects are fully taken into account, the interpretation is not as simple, but evidently the contribution tied to $\chi_{i j}$ continues to dominate behavior.

It is also interesting to note that the one-parameter version (41) has $(1 / 3) \ell_{*}^{2} \dot{\alpha}^{2}$ as its contribution to $\dot{E}_{\mathrm{P}}^{2}$ for the classical limit noted above. Roughly similar gradient effects from the two versions would be expected when $\ell_{*}=2 \sqrt{3} \ell_{2}$, and this is seen to be the case when the two sets of results in Figs. 3 and 4 are compared. The factor $2 \sqrt{3}$ simply reflects the particular manner in which the invariants multiplying the respective length parameters have been normalized. Nevertheless, the "correspondence" factors are strongly problem dependent. For example, for the shear problem in the previous subsection, the two formulations are identical when $\ell_{*}=\ell_{2}$ if $\ell_{1}=\ell_{3}=0$. If each of the two formulations were to be calibrated by the shear problem such that $\ell_{*}=\ell_{2}$ (with $\ell_{1}=\ell_{3}=0$ ), then at least one of the versions must clearly be significantly in error in predicting wire torsion. This strong problem dependence of the size effect is the essence underlying the necessity of the multiple parameter formulation, as will be further reinforced by a study of void growth.

Solutions to the wire torsion problem were also generated based on the deformation theory functional (29) for precisely the same input parameters and the same RambergOsgood stress-strain curve. A similar finite element formulation was used with Newton iteration to provide convergence at arbitrary twist. The solutions obtained are almost identical to those plotted in Fig. 4: the two sets of predictions are essentially indistinguishable from one another when the respective plots are compared, and therefore the deformation theory results have not been displayed. Given the pure power nature of the stress-strain curve when the plastic strains dominate the elastic strains, it is not 
surprising that the agreement should be good at sufficiently large twist. Proportional stressing is satisfied asymptotically for large twist. The fact that the agreement is excellent over the entire range of twist is undoubtedly due to the fact that the deformation theory solution interpolates between two limits (e.g. small and large twist) for which it exactly reproduces the flow theory solution.

\subsection{Size effects in void growth}

Stretch gradients play a dominant role in void growth and indentation. Here the simplest void growth problem is considered which captures the essence of the strain gradient phenomenon. The growth of an isolated spherical void is analyzed; it has an initial radius $R$ and resides in an infinite solid subject to a remote spherically symmetric tension $\sigma_{\infty}$. A small strain formulation is invoked and the material is taken to be elastically incompressible and characterized by the Ramberg-Osgood tensile stress-strain curve (32). The solution is spherically symmetric and a standard spherical coordinate system is used with $r$ as the distance from the origin at the center of the void. Elastic (and plastic) incompressibility implies that the displacement rate is specified according to $\dot{u}_{r}=\dot{A} r^{-2}$, where $\dot{A}$ is an amplitude factor related to the volume expansion rate of the void by $\dot{V}=4 \pi \dot{A}$. The total strain rates are given by $\dot{\varepsilon}_{r r}=-2 \dot{\varepsilon}_{\theta \theta}=-2 \dot{\varepsilon}_{\phi \phi}=-2 \dot{A} r^{-3}$. The nonzero plastic strain rate components are given by $\dot{\varepsilon}_{r r}^{\mathrm{P}}=-2 \dot{\varepsilon}_{\theta \theta}^{\mathrm{P}}=-2 \dot{\varepsilon}_{\phi \phi}^{\mathrm{P}}=-\dot{\varepsilon}_{\mathrm{P}}$ where $\dot{\varepsilon}_{\mathrm{P}}(r)$ is the unknown distribution of the plastic strain rate.

As in the case of wire torsion, the plastic strain rate has both spatially varying amplitude and direction. The nonzero components of the gradient of plastic strain rate are

$$
\rho_{r r r}=-\dot{\varepsilon}_{\mathrm{P}}^{\prime}, \quad \rho_{\theta \theta r}=\rho_{\phi \phi r}=\dot{\varepsilon}_{\mathrm{P}}^{\prime} / 2, \quad \rho_{r \theta \theta}=\rho_{\theta r \theta}=\rho_{r \phi \phi}=\rho_{\phi r \phi}=-3 \dot{\varepsilon}_{\mathrm{P}} / 2,
$$

The gradient-dependent effective plastic strain rate in (10) is found to be

$$
\dot{E}_{\mathrm{P}}^{2}=\dot{\varepsilon}_{\mathrm{P}}^{2}+\frac{9}{10} \ell_{1}^{2}\left(\dot{\varepsilon}_{\mathrm{P}}^{\prime}-2 r^{-1} \dot{\varepsilon}_{\mathrm{P}}\right)^{2}+\frac{8}{5} \ell_{3}^{2}\left(\dot{\varepsilon}_{\mathrm{P}}^{\prime}+3 r^{-1} \dot{\varepsilon}_{\mathrm{P}}\right)^{2}
$$

with no dependence on $\ell_{2}$. In contrast, the measure for the one-parameter theory (1) is

$$
\dot{E}_{\mathrm{P}}^{2}=\dot{\varepsilon}_{\mathrm{P}}^{2}+\ell_{*}^{2} \dot{\varepsilon}_{\mathrm{P}}^{2}
$$

Functional (21) governing the variational statement of the incremental problem becomes

$$
I\left(\dot{\varepsilon}_{\mathrm{P}}\right)=\frac{1}{2} \int_{R}^{\infty}\left\{E\left(-2 \dot{A} r^{-3}+\dot{\varepsilon}_{\mathrm{P}}\right)^{2}+h\left(E_{\mathrm{P}}\right) \dot{E}_{\mathrm{P}}^{2}\right\} 4 \pi r^{2} \mathrm{~d} r .
$$

Plastic flow is assumed to be unconstrained at the surface of the void with the consequence that the natural boundary condition there is $\dot{\tau}_{i} n_{i}=0$. The most convenient way to determine the relation between the remote stress rate, $\dot{\sigma}_{\infty}$, and $\dot{A}$ in a numerical computation of the type conducted here is to make use of the identity, $2 \pi \dot{\sigma}_{\infty} \dot{A}=I\left(\dot{\varepsilon}_{\mathrm{P}}\right)$, where $I$ is evaluated after the solution, $\dot{\varepsilon}_{\mathrm{P}}$, has been obtained. 


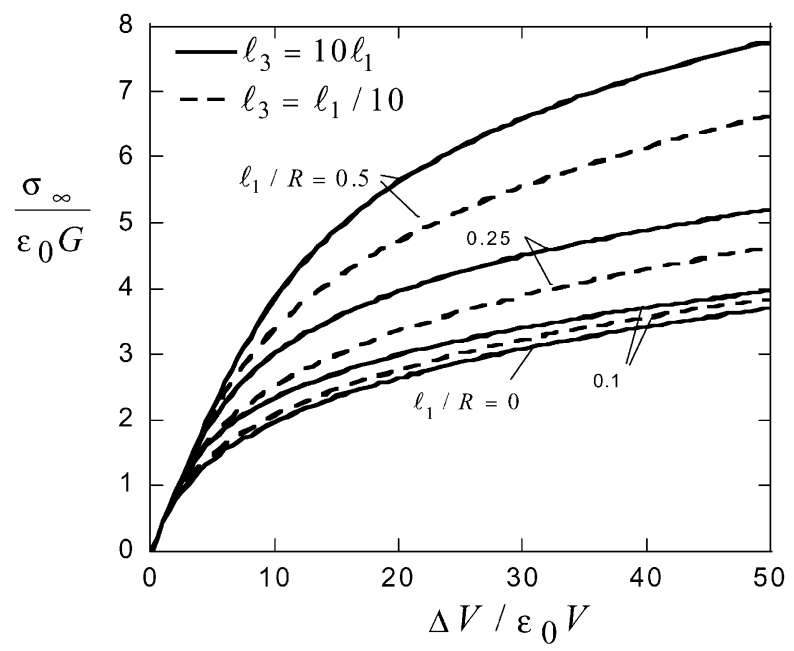

Fig. 5. Remote stress as a function of normalized volume expansion based on the general formulation for a spherical void subject to hydrostatic tension, $\sigma_{\infty}$, at infinity with $N=0.2$ and elastic incompressibility. The initial void radius is $R$. The length parameter $\ell_{1}$ is the most important in determining the strain gradient effect in void growth; $\ell_{3}$ has very little influence, and $\ell_{2}$ does not enter the problem. The calculation maps the region exterior to the void onto $(0,1)$ and employs 100 elements in the mapped region. The calculation attains $\left(\Delta V / \varepsilon_{0}\right) / V_{0}=50$ in 500 incremental steps.

Numerical solutions for each of the formulations were generated in a manner similar to that used for wire torsion, except that in this case the region exterior to the void is mapped onto $(0,1)$ prior to discretization. With $\Delta V$ as the volume expansion of the void and $V_{0}$ as its initial volume, the normalized volume expansion is taken as $\left(\Delta V / \varepsilon_{0}\right) / V_{0}$. Curves of remote stress versus volume expansion for the general formulation based on (44) are given in Fig. 5. It is seen that the length parameter $\ell_{1}$ is far more important that $\ell_{3}$ in setting the influence of strain gradients on void growth. Indeed, changing $\ell_{3}$ by a factor of 10 , either larger or smaller than $\ell_{1}$, has relatively little consequence. Qualitatively, one can see why this might be from the fact that the spherically symmetric expansion involves only stretch gradients and no rotation gradients. Where plastic strains are large compared to elastic strains incompressibility dictates $\dot{\varepsilon}_{\mathrm{P}} \cong 2 \dot{A} r^{-3}$, such that the contribution to $\dot{E}_{\mathrm{P}}$ in (44) from $\ell_{3}$ vanishes and, more fundamentally, $\chi_{i j}=0$. The corresponding void growth behavior as predicted by the one-parameter formulation based on (45) is shown in Fig. 6. The parameter $\ell_{*}$ has roughly the same influence as $\ell_{1}$ in the multiple parameter formulation, apart from a factor of about 2 .

Numerical solutions for the deformation theory formulation based on (28) and (29) were also produced for the void growth problem. The results are in close agreement with the corresponding flow theory predictions in Fig. 5. The maximum discrepancy is larger than in the case of wire torsion, but the discrepancy in remote stress is still less than $10 \%$ at any given void expansion. 


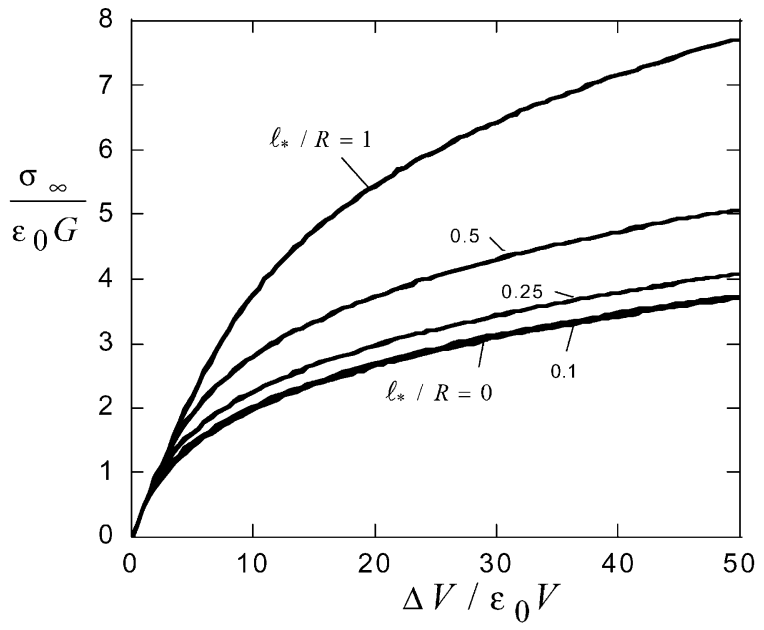

Fig. 6. Remote stress as a function of normalized volume expansion based on the one-parameter formulation for a spherical void subject to hydrostatic tension, $\sigma_{\infty}$, at infinity with $N=0.2$ and elastic incompressibility.

\section{Implications from experimental observations}

According to the general formulation, size effects in wire torsion are controlled by $\ell_{2}$, while $\ell_{1}$ is the important length parameter in void growth. Indentation is similar to void growth in that stretch gradients are dominant with $\ell_{1}$ playing the primary role in setting size effects in hardness testing. When $\ell_{2}$ is chosen to give a best fit to data on the torsion of fine copper wires, Fleck et al. (1994) obtained $\ell_{2} \cong 2 \mu \mathrm{m}$. ${ }^{3}$ Begley and Hutchinson (1998) analyzed the size dependence of indentation data for different metals of varying hardness to infer that $\ell_{1}$ usually fell within the range between 0.2 and $0.5 \mu \mathrm{m}$. Both efforts were based on the theory of Fleck and Hutchinson (1997), but this theory gives quantitatively similar predictions for the plastic responses as the present multiple parameter formulation, as will be discussed in the next section. The difference between these two length parameters is highly significant from a quantitative standpoint. For example, if one took $\ell_{1}$ to be $2 \mu \mathrm{m}$, one would over-predict the size effect in indentation by almost an order of magnitude for most metals. Moreover, if $\ell_{1}$ were as large as $2 \mu \mathrm{m}$, one would conclude that voids with diameters as small as about $10 \mu \mathrm{m}$ should already display a strong size effect (cf. Fig. 5). While there is no firm experimental data for the void diameter at which size effects should set in, there is ample anecdotal evidence that the transitional diameter must be substantially smaller than $10 \mu \mathrm{m}$. Voids involved in the ductile fracture of many metals are typically observed to be from a micron to several microns in diameter prior to their final enlargement. Appropriately or not, void growth in ductile fracture is routinely modeled

\footnotetext{
${ }^{3}$ Fleck et al. (1994) employed another normalization with a single length parameter, $\ell_{\mathrm{CS}}=2 \ell_{2}($ see ahead to Eq. (47)). They obtained $\ell_{\mathrm{CS}} \cong 4 \mu \mathrm{m}$ in their fit to the wire torsion data.
} 
and analyzed using conventional plasticity theory. If, however, $\ell_{1}$ is in the range from 0.2 to $0.5 \mu \mathrm{m}$ as inferred from the indentation data, then from Fig. 5 it follows that the transitional void diameter at which size effects become important would be on the order of $1 \mu \mathrm{m}$. Experimental observations of the substantial resistance to void growth predicted for voids smaller than this transitional size should be possible, but to our knowledge none have been reported.

The distinct difference between the size effects in wire torsion, indentation and void growth is problematic for a one-parameter formulation. For example, if one uses the theoretical predictions in Fig. 4 to fit the wire torsion data of Fleck et al. (1994), one finds that $\ell_{*}$ must be at least as large as $12 \mu \mathrm{m}$. Then, if $\ell_{*}=12 \mu \mathrm{m}$ is used in conjunction with Fig. 6 to predict void growth, one would conclude that voids with diameters less than $25 \mu \mathrm{m}$ should display a large size effect. Presumably, the conclusion drawn for indentation would be equally erroneous if theoretical predictions were available to make a comparison.

The argument outlined above for the necessity of more than one length parameter in any phenomenological theory generalizing $J_{2}$ flow theory to include strain gradients appears to be compelling. It seems highly unlikely that the various phenomena reviewed could be subsumed under a theory that invokes only one length parameter. In an attempt to reduce the number of necessary length parameters from three to two, Begley and Hutchinson (1998) proposed to neglect the contributions of the invariant quantity, $\chi_{i j} \chi_{j i}$, in (6) and (10). This invariant appears to play a relatively limited role in most of the problems that have been investigated to date. With

$$
\ell_{2}=\frac{1}{2} \ell_{\mathrm{CS}}, \quad \ell_{3}=\sqrt{\frac{5}{24}} \ell_{\mathrm{CS}},
$$

the effective plastic strain rate (10) becomes

$$
\dot{E}_{\mathrm{P}}^{2}=\dot{\varepsilon}_{\mathrm{P}}^{2}+\ell_{1}^{2} \eta_{i j k}^{(1)} \eta_{i j k}^{(1)}+\frac{2}{3} \ell_{\mathrm{CS}}^{2} \chi_{i j} \chi_{i j}
$$

Here, $\ell_{\mathrm{CS}}$ is the length parameter originally introduced by Fleck et al. (1994) in a special couple stress formulation (with $\ell_{1}=0$ ) tied exclusively to gradients of rotations. If this reduction in the set of length parameters survives upon exposure to further experimental data, it follows that the length parameters for representative metals are

$$
\ell_{1}=0.2-0.5 \mu \mathrm{m}, \quad \ell_{2} \cong 2 \mu \mathrm{m}, \quad\left(\ell_{3}=\sqrt{5 / 6} \ell_{2}, \ell_{\mathrm{CS}}=2 \ell_{2}\right)
$$

The pure bending tests of thin nickel sheets of Stolken and Evans (1998) provide further experimental support for (49). In pure bending, the conventional effective plastic strain rate, $\dot{\varepsilon}_{\mathrm{P}}$, varies through the thickness of the sheet. Its distribution completely specifies the plastic deformation. The effective plastic strain rate (10) is

$$
\dot{E}_{\mathrm{P}}^{2}=\dot{\varepsilon}_{\mathrm{P}}^{2}+\ell^{2} \dot{\varepsilon}_{\mathrm{P}}^{\prime 2}
$$

with

$$
\ell^{2} \equiv \frac{4}{5} \ell_{1}^{2}+\ell_{2}^{2}+\frac{6}{5} \ell_{3}^{2}
$$

Stolken and Evans used the Fleck-Hutchinson deformation theory (see the next section) to analyze pure bending and found that the length parameters specified in (49) gave a reasonable fit to their data for nickel sheets. Bending is like torsion in that the 
contribution from $\ell_{1}$ is relatively unimportant given the values in (49). In principle, it should be possible to separate the roles of $\ell_{2}$ and $\ell_{3}$ using data from bending and torsion, because torsion depends only on $\ell_{2}$, but that is not yet possible using existing data. It is interesting to note that the combination of the length parameters that governs bending is the same as that in pure shear (compare (51) and (36)).

\section{Relation of the present theory to previous higher order theories}

Deformation theory and flow theory extensions of $J_{2}$ theory to account for strain gradient effects were proposed earlier by Fleck and Hutchinson (1997). These formulations make use of a strain gradient decomposition with multiple length parameters similar to that employed in the generalization proposed here. The deformation theory version has the virtue that it is relatively simple to apply, and for basic problems like those considered here it is amenable to closed-form solution. Two such examples will be used to illustrate that the strain gradient effects predicted by the earlier theory of Fleck and Hutchinson are quantitatively similar to those of the present class of theories.

In the deformation theory version for an incompressible solid (Fleck and Hutchinson, 1997), the gradients of the total strains are presented by the third-order tensor $\eta_{i j k}=$ $\eta_{j i k} \equiv u_{k, i j}=\varepsilon_{k i, j}+\varepsilon_{k j, i}-\varepsilon_{i j, k}$. Then, the orthogonal decomposition cited in Section 2 is used to form an isotropic overall effective strain measure according to

$$
E_{\mathrm{e}}^{2}=\varepsilon_{\mathrm{e}}^{2}+\ell_{1}^{2} \eta_{i j k}^{(1)} \eta_{i j k}^{(1)}+\ell_{2}^{2} \eta_{i j k}^{(2)} \eta_{i j k}^{(2)}+\ell_{3}^{2} \eta_{i j k}^{(3)} \eta_{i j k}^{(3)},
$$

where $\varepsilon_{\mathrm{e}}=\sqrt{2 \varepsilon_{i j} \varepsilon_{i j} / 3}$ is the effective total strain and the $\ell$ 's are introduced analogously to those in (10). The material is a small strain, nonlinear elastic solid which falls within the class of higher-order theories investigated by Toupin (1962) and Mindlin (1964). Let $W\left(\varepsilon_{\mathrm{e}}\right)=\int_{0}^{\varepsilon_{\mathrm{e}}} \sigma(\tilde{\varepsilon}) \mathrm{d} \tilde{\varepsilon}$ be the strain energy density of the solid in the gradient-independent limit (i.e. the incompressible $J_{2}$ deformation theory solid with vanishing $\ell$ 's), with $\sigma(\varepsilon)$ now as the tensile data for stress as a function of total uniaxial strain. The energy density of the generalized deformation theory is taken to be $W\left(E_{\mathrm{e}}\right)$. The gradient-dependent version of the flow theory proposed by Fleck and Hutchinson has been constructed such that it coincides with the deformation theory for proportional stressing.

In wire torsion, the one nonzero strain component is $\gamma_{\theta}=\alpha r$ and the effective strain (52) is $E_{\mathrm{e}}^{2}=\alpha^{2} r^{2} / 3+4 \alpha^{2} \ell_{2}^{2}$, involving only the one length parameter. The energy density $W\left(E_{\mathrm{e}}\right)$ is evaluated using the Ramberg-Osgood tensile curve (32). The relation between the normalized torque and twist from the deformation theory can be obtained in closed form as

$$
\frac{T}{G \varepsilon_{0} R^{3}}=\frac{2 \pi E}{G} \int_{0}^{1} \mu\left(x^{2} / 3+4\left(\ell_{2} / R\right)^{2}\right)^{1 / 2} x \mathrm{~d} x,
$$

where $\mu$ satisfies $\mu+\mu^{n}=\left(\alpha R / \varepsilon_{0}\right)\left(x^{2} / 3+4\left(\ell_{2} / R\right)^{2}\right)^{1 / 2}$. Predictions from (53) are plotted in Fig. 7. They can compared directly with the predictions from the new formulation in Fig. 3. The simple deformation theory correctly extracts the dominant role of $\ell_{2}$. Moreover, apart from the differences in behavior at low twist, the two sets of predictions 


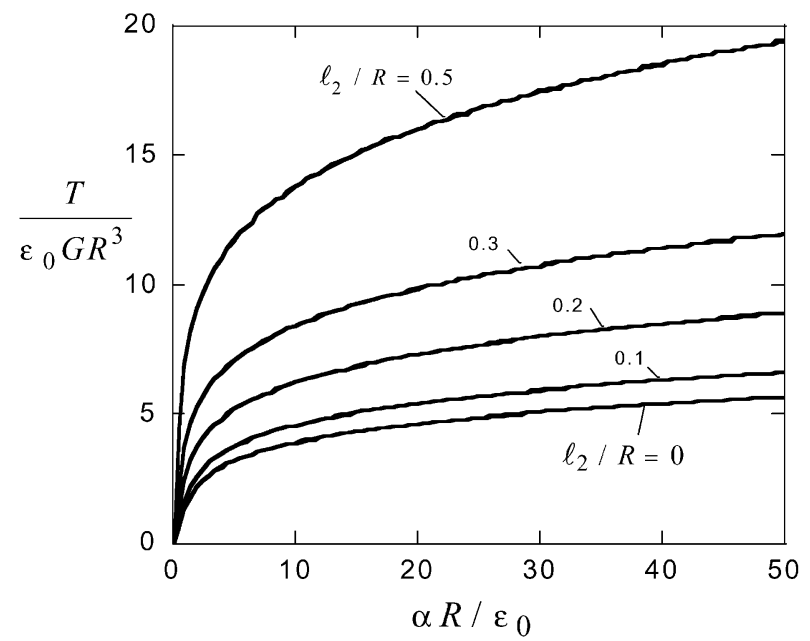

Fig. 7. Torque versus twist based on the deformation theory of Fleck and Hutchinson (1997) for a solid wire of radius $R$ with $N=0.2$ and $v=0.3$. These results can be compared with the results computed using the new formulation in Fig. 3. (Elastic compressibility of the wire does not play a role in the solution, however the value of Poisson's ratio enters into the normalization used when evaluating $G / E$.)

are quantitatively similar. The previous Fleck-Hutchinson (1997) deformation theory formulation incorrectly introduces a dependence on strain gradients in the linear elastic range, producing the unphysical dependence of the initial slope of the torque-twist relation on $\ell_{2}$. Thus, this discrepancy between the two theories is due to the limitations of the earlier Fleck-Hutchinson deformation theory and not the new formulation. As the plastic strains become large compared to the elastic strains, the solutions to earlier deformation theory become asymptotic to solutions to the new deformation theory. The void growth problem can also be solved in closed form using the earlier deformation theory. It similarly captures all the important details of the behavior in Fig. 5, apart from the behavior in the elastic range. In the limit of a material with elasticity suppressed, solutions from the two deformation theories can be shown to coincide for a fairly wide class of boundary conditions, although not all. This connection underlies the relatively close agreement expected for a wide spectrum of problems.

\section{Summary}

A class of phenomenological strain gradient plasticity theories is formulated to accommodate up to three material length scales. A special case involves a single length parameter, and is of similar form to that proposed by Aifantis and co-workers (Aifantis, 1984; Muhlhaus and Aifantis, 1991). The necessity of including a dependence on at least two length parameters in any extension of classical $J_{2}$ plasticity theory to account for size effects at the micron scale has been laid out in the current treatment. The new 
generalized versions give predictions in the plastic range that are quantitatively similar to the earlier theories proposed by Fleck and Hutchinson (1997). However, the new versions have clear advantages over the earlier theories in numerical implementation and in representation of elastic behavior.

Both the flow and deformation versions of the new formulation employ the displacement components and plastic strain amplitude as the primary variables in the variational statement of boundary value problems. This aspect accounts for the advantages of this class of formulations in framing finite element representations for numerical solutions. Only the primary variables and their first gradients enter the variational statement of the problems. For most higher-order theories, such as that of Fleck and Hutchinson (1997), the primary variables are the displacement components, but both their first and second gradients enter the variational statement, significantly increasing the difficulty of developing an accurate finite element representation. The version of the theory of the type originally suggested by Aifantis and coworkers, based on the effective strain rate (1) with one length parameter, is the simplest to implement in a finite element framework. The generalized versions are somewhat more complicated to implement because the stress direction, $m_{i j}$, and its gradient must be evaluated at each point to determine the coefficients $A_{i j}, B_{i}$ and $C$ in (20) or (28). However, these can be evaluated in the current state (or at the current state of a sequence of iterations in the case of the deformation theory), and therefore do not require the introduction of higher-order elements for the solution variables in the incremental or iterative problem.

Another distinct advantage of the new class of theories over the earlier FleckHutchinson theories is the representation of behavior in the elastic range. Use of the plastic strains as primary variables leads naturally to a formulation with conventional behavior in the elastic range. By contrast, length parameters are artificially present in the elastic range in the earlier Fleck-Hutchinson theories, as illustrated by the deformation theory solutions in the previous section. In the flow theory version, the length parameters in the elastic range can be set independently from those in the plastic range, but they cannot be taken to be zero. The earlier Fleck-Hutchinson deformation theory formulation has an advantage over the new theory in that its simplicity permits closed-form solutions to basic problems. It should continue to have a useful role to play given its relationship with the new deformation theory when elasticity is neglected, and given the close connection between deformation and flow theory solutions for many problems of interest.

An effort has been made to reveal the higher-order nature of the new theories as clearly as possible. The existence of the higher-order stress, $\tau_{i}$, requires some change to the conventional interpretation of the field equations. Equally important is the latitude the higher-order theories offer to model nonstandard boundary and interface conditions, as dictated on physical grounds. Illustrations are given in the paper for both constrained and unconstrained plastic deformation at a boundary. Many examples of nonstandard conditions for thin films, multiple phase composites and other metal systems can be enumerated. This appears to be an important aspect of plasticity at the small scale. Thus, if continuum theories are to be used to predict elastic-plastic behavior at the micron scale, a higher-order theory is likely to be required. 


\section{Acknowledgements}

The work of JWH was supported in part by the Danish Research Agency, by ONR Grant No. 0014-96-1-1028, by NSF grant DMR-94-00396 and by the Division of Engineering and Applied Sciences, Harvard University. The work of NAF was supported by Cambridge University.

\section{Appendix A.}

\section{A.1. Expressions for $A_{i j}, B_{i}$ and $C$}

Examples of the expression of $\dot{E}_{\mathrm{P}}^{2}$ for specific problems are given in the body of the paper. For the general case, in Cartesian coordinates with $\rho_{i j k}$ given by (19), the coefficients in (20) and in (28) are given by

$$
\begin{aligned}
& A_{i j}=\ell_{1}^{2}\left\{\frac{1}{2} \delta_{i j}+\frac{2}{5} m_{i p} m_{j p}\right\}+e_{p i r} m_{q r}\left\{L_{2}^{2} e_{p j v} m_{q v}+L_{3}^{2} e_{q j v} m_{p v}\right\}, \\
& B_{i}=\ell_{1}^{2}\left\{\frac{4}{3} m_{p q} m_{p i, q}-\frac{8}{15} m_{i p} m_{p q, q}\right\}+2 e_{p i r} m_{q r}\left\{L_{2}^{2} e_{p u v} m_{q v, u}+L_{3}^{2} e_{q u v} m_{p v, u}\right\}, \\
& C=\ell_{1}^{2}\left\{\frac{1}{3} m_{i j, k}\left(m_{i j, k}+2 m_{j k, i}\right)-\frac{4}{15} m_{k i, i} m_{k j, j}\right\}+e_{p i r} m_{q r, i}\left\{L_{2}^{2} e_{p u v} m_{q v, u}+L_{3}^{2} e_{q u v} m_{p v, u}\right\}
\end{aligned}
$$

with $L_{2}^{2}=\frac{4}{3} \ell_{2}^{2}+\frac{8}{5} \ell_{3}^{2}$ and $L_{3}^{2}=\frac{4}{3} \ell_{2}^{2}-\frac{8}{5} \ell_{3}^{2}$. Further reduction can be obtained noting that

$$
\begin{aligned}
& e_{p i r} e_{p j v} m_{q r} m_{q v}=\frac{3}{2} \delta_{i j}-m_{q i} m_{q j}, \quad e_{p i r} e_{p u v} m_{q r} m_{q v, u}=-m_{q r} m_{q i, r}, \\
& e_{p i r} e_{p u v} m_{q r, i} m_{q v, u}=m_{q r, u} m_{q r, u}-m_{q r, v} m_{q v, r} .
\end{aligned}
$$

\section{A.2. Minimum and uniqueness principles for flow theory solid}

We begin by showing that the plastic dissipation within the strain gradient flow theory solid of Section 3.1 is positive. The internal plastic work rate can be written from (12) and (15) as

$$
\text { Internal plastic work }=\int_{V}\left[\sigma_{\mathrm{e}} \delta \varepsilon_{\mathrm{P}}+\left(\tau_{i} \delta \varepsilon_{\mathrm{P}}\right)_{, i}\right] \mathrm{d} V
$$

and re-interpreted as follows. The first term, $\sigma_{\mathrm{e}} \delta \varepsilon_{\mathrm{P}}$, is the plastic dissipation, and is never negative. The volume integral of the second term $\left(\tau_{i} \delta \varepsilon_{\mathrm{P}}\right)_{, i}$ equals the prescribed surface plastic work $\int_{S}\left[n_{i} \tau_{i} \delta \varepsilon_{\mathrm{P}}\right] \mathrm{d} S$ by the divergence theorem, and may be positive or negative.

The strain gradient version of $J_{2}$ flow theory satisfies the generalized form of Drucker's stability postulates (Drucker, 1951) provided $h>0$ :

$$
\dot{Q} \dot{\varepsilon}_{\mathrm{P}}+\dot{\tau}_{i} \dot{\varepsilon}_{\mathrm{P}, i}=h \dot{E}_{\mathrm{P}}^{2} \geqslant 0
$$


for a stress rate $\left(\dot{Q}, \dot{\tau}_{i}\right)$ corresponding to any strain rate $\left(\dot{\varepsilon}_{\mathrm{P}}, \dot{\varepsilon}_{\mathrm{P}, i}\right)$, and

$$
\left(\sigma_{i j}-\sigma_{i j}^{*}\right) \dot{\varepsilon}_{i j}^{\mathrm{P}} \geqslant 0
$$

for a stress state $\sigma_{i j}$ associated with a plastic strain rate $\dot{\varepsilon}_{i j}^{\mathrm{P}}$, and a neighboring stress state $\sigma_{i j}^{*}$ within or on the yield surface.

Minimum principle (21) for the displacement rate follows directly from those outlined by Koiter (1960) and Hill (1966), as is restated as

$$
\begin{aligned}
I\left(\dot{u}_{i}, \dot{\varepsilon}_{\mathrm{P}}\right)= & \frac{1}{2} \int_{V}\left\{C_{i j k l}\left(\dot{\varepsilon}_{i j}-\dot{\varepsilon}_{\mathrm{P}} m_{i j}\right)\left(\dot{\varepsilon}_{k l}-\dot{\varepsilon}_{\mathrm{P}} m_{k l}\right)+h\left(E_{\mathrm{P}}\right) \dot{E}_{\mathrm{P}}^{2}\right\} \mathrm{d} V \\
& -\int_{S_{T}}\left(\dot{T}_{i}^{0} \dot{u}_{i}+\dot{t}^{0} \dot{\varepsilon}_{\mathrm{P}}\right) \mathrm{d} S
\end{aligned}
$$

where $\dot{T}_{i}^{0}$ and $\dot{t}^{0}$ are prescribed traction rates on $S_{T}$, and $E_{\mathrm{P}}=\int \dot{E}_{\mathrm{P}} \mathrm{d} t$ is the accumulated effective plastic strain. Let $\left(\dot{u}_{i}, \dot{\varepsilon}_{\mathrm{P}}\right)$ be the exact solution, and $\left(\dot{u}_{i}^{*}, \dot{\varepsilon}_{\mathrm{P}}^{*}\right)$ be any kinematically admissible field. Then, $I\left(\dot{u}_{i}^{*}, \dot{\varepsilon}_{\mathrm{P}}^{*}\right)$ achieves a minimum value by the actual solution.

Proof. Denote the difference in displacement rates by $\Delta \dot{u}_{i} \equiv \dot{u}_{i}^{*}-\dot{u}_{i}$, and likewise for the strain rate and stress rate quantities. Then, direct evaluation of $\Delta I=I\left(\dot{u}_{i}^{*}, \dot{\varepsilon}_{\mathrm{P}}^{*}\right)-$ $I\left(\dot{u}_{i}, \dot{\varepsilon}_{\mathrm{P}}\right)$ gives

$$
\begin{aligned}
\Delta I= & \frac{1}{2} \int_{V}\left[C_{i j k l}\left(\Delta \dot{\varepsilon}_{i j}-\Delta \dot{\varepsilon}_{\mathrm{P}} m_{i j}\right)\left(\Delta \dot{\varepsilon}_{k l}-\Delta \dot{\varepsilon}_{\mathrm{P}} m_{k l}\right)\right] \mathrm{d} V \\
& +\frac{1}{2} \int_{V}\left[\dot{Q}^{*} \dot{\varepsilon}_{\mathrm{P}}^{*}+\dot{Q} \dot{\varepsilon}_{\mathrm{P}}-2 \dot{Q}_{\dot{\varepsilon}_{\mathrm{P}}^{*}}^{*}+\dot{\tau}_{i}^{*} \dot{\varepsilon}_{\mathrm{P}, i}^{*}+\dot{\tau}_{i} \dot{\varepsilon}_{\mathrm{P}, i}-2 \dot{\tau}_{i} \dot{\varepsilon}_{\mathrm{P}, i}^{*}\right] \mathrm{d} V .
\end{aligned}
$$

Now, the first integrand on the right-hand side of (A.6) is positive since the elastic stiffness $C_{i j k l}$ is taken to be positive definite. It can be shown that the second integrand on the right-hand side of (A.6) satisfies the inequality

$$
\dot{Q}^{*} \dot{\varepsilon}_{\mathrm{P}}^{*}+\dot{Q} \dot{\varepsilon}_{\mathrm{P}}-2 \dot{Q} \dot{\varepsilon}_{\mathrm{P}}^{*}+\dot{\tau}_{i}^{*} \dot{\varepsilon}_{\mathrm{P}, i}^{*}+\dot{\tau}_{i} \dot{\varepsilon}_{\mathrm{P}, i}-2 \dot{\tau}_{i} \dot{\varepsilon}_{\mathrm{P}, i}^{*} \geqslant h \Delta \dot{E}_{\mathrm{P}} \Delta \dot{E}_{\mathrm{P}} \geqslant 0
$$

for the four possible loading cases of plastic loading and unloading for each of the two solutions. Provided $h$ is assumed to be positive, we conclude that $\Delta I>0$ for all trial solutions. The exact solution minimizes $I$, and the exact solution is unique since the minimum is absolute.

Next, we prove uniqueness of stress rate for any assumed strain rate, and vice versa, again for the case $h>0$. The proof follows that of Hill (1966) for multislip of elastic-plastic crystals. Let $\dot{\sigma}_{i j}$ and $\dot{\sigma}_{i j}^{*}$ be two stress rates with associated strain rates $\dot{\varepsilon}_{i j}$ and $\dot{\varepsilon}_{i j}^{*}$; the associated plastic strain rates $\dot{\varepsilon}_{\mathrm{P}}$ and $\dot{\varepsilon}_{\mathrm{P}}^{*}$ may or may not vanish. Then, upon noting that

$$
\Delta \dot{\sigma}_{i j} \Delta \dot{\varepsilon}_{i j}^{\mathrm{e}}+\Delta \dot{Q} \Delta \dot{\varepsilon}_{\mathrm{P}}+\Delta \dot{\tau}_{i} \Delta \dot{\varepsilon}_{\mathrm{P}, i} \geqslant C_{i j k l} \Delta \dot{\varepsilon}_{i j}^{\mathrm{e}} \Delta \dot{\varepsilon}_{k l}^{\mathrm{e}}+h \Delta \dot{E}_{\mathrm{P}} \Delta \dot{E}_{\mathrm{P}} \geqslant 0,
$$


we conclude that $\dot{\varepsilon}_{i j}$ and $\dot{\varepsilon}_{\mathrm{P}}$ are unique if $\dot{\sigma}_{i j}$ is given, and that $\dot{\sigma}_{i j}$ is unique if $\dot{\varepsilon}_{i j}$ and $\dot{\varepsilon}_{\mathrm{P}}$ are given.

\section{References}

Acharya, A., Bassani, J.L., 2000. Incompatibility and crystal plasticity. Mech. Phys. Solids 48, 1565-1595. Aifantis, E.C., 1984. On the microstructural origin of certain inelastic models. Trans. ASME J. Eng. Mat. Tech. 106, 326-330.

Aifantis, E.C., 1999. Strain gradient interpretation of size effects. Int. J. Fracture 95, $299-314$.

Arsenlis, A., Parks, D.M., 1999. Crystallographic aspects of geometrically-necessary and statistically-stored dislocation density. Acta Mater. 47, 1597-1611.

Bassani, J.L., Needleman, A., Van der Giessen, E., 2001. Plastic flow in a composite: a comparison of nonlocal continuum and discrete dislocation predictions. Int. J. Solids Structs. 38, 833-853.

Begley, M.R., Hutchinson, J.W., 1998. The mechanics of size-dependent indentation. J. Mech. Phys. Solids 46, 2049-2068.

Budiansky, B., 1959. A reassessment of deformation theories of plasticity. J. Appl. Mech. 26, 259-264.

Busso, E.P., Meissonneir, F.T., O'Dowd, N.P., 2000. Gradient-dependent deformation of two-phase single crystals. J. Mech. Phys. Solids 48, 2333-2362.

de Borst, R., Muhlhaus, H.-B., 1992. Gradient-dependent plasticity: formulation and algorithmic aspects. Int. J. Numer. Meth. Eng. 35, 521-539.

Drucker, D.C., 1951. A more fundamental approach to plastic stress-strain relations. Proceedings of the 1st US National Congress Applied Mechanics, ASME, New York, pp. 487-491.

Fleck, N.A., Hutchinson, J.W., 1997. Strain gradient plasticity. In: Hutchinson, J.W., Wu, T.Y. (Eds.), Advances in Applied Mechanics, Vol. 33. Academic Press, New York, pp. 295-361.

Fleck, N.A., Muller, G.M., Ashby, M.F., Hutchinson, J.W., 1994. Strain gradient plasticity: theory and experiment. Acta Metall. Mater. 42, 475-487.

Gurtin, M.E., 2000. On the plasticity of single crystals: free energy, microforces plastic-strain gradients. J. Mech. Phys. Solids 48, 989-1036.

Hill, R., 1966. Generalized constitutive relations for incremental deformation of metal crystals by multislip. J. Mech. Phys. Solids 14, 95-102.

Koiter, W.T., 1960. General theorems for elastic-plastic solids. In: Sneddon, I.N., Hill, R. (Eds.), Progress in Solids Mechanics, Vol. 1. North-Holland, Amsterdam, pp. 167-221.

Mikkelsen, L.P., 1999. Necking in rectangular tensile bars approximated by 2-D gradient dependent plasticity model. Eur. J. Mech. A/Solids 18, 805-818.

Mindlin, R.D., 1964. Micro-structure in linear elasticity. Arch. Rational Mech. Anal. 16, 51-78.

Muhlhaus, H-B., Aifantis, E.C., 1991. A variational principle for gradient plasticity. Int. J. Solids Struct. 28, $845-857$.

Nix, W.D., Gao, H., 1998. Indentation size effects in crystalline materials: a law for strain gradient plasticity. J. Mech. Phys. Solids 46, 411-425.

Shu, J.Y., Fleck, N.A., 1998. The prediction of a size effect in micro-indentation. Int. J. Solids Struct. 35, $1363-1383$.

Shu, J.Y., Fleck, N.A., 1999. Strain gradient crystal plasticity: size-dependent deformation of bicrystals. J. Mech. Phys. Solids 47, 297-324.

Shu, J.Y., Fleck, N.A., Van der Giessen, E., Needleman, A., 2001. Boundary layers in constrained plastic flow: comparison of nonlocal and discrete dislocation plasticity. J. Mech. Phys. Solids, to appear.

Sluys, L.J., Estrin, Y., 2000. The analysis of shear banding with a dislocation based gradient plasticity model. 37, 7127-7142.

Smyshlyaev, V.P., Fleck, N.A., 1994. Bounds and estimates for the overall plastic behaviour of composites with strain gradient effects. Proc. Roy. Soc. A451, 795-810.

Smyshlyaev, V.P., Fleck, N.A., 1996. The role of strain gradients in the grain size effect for polycrystals. J. Mech. Phys. Solids 44, 465-495.

Stolken, J.S., Evans, A.G., 1998. A microbend test method for measuring the plasticity length scale. Acta Mater. 46, 5109-5115. 
Toupin, R.A., 1962. Elastic materials with couple stresses. Arch. Rational Mech. Anal. 11, 385-414.

Wei, Y., Hutchinson, J.W., 1997. Steady-state crack growth and work of fracture for solids characterized by strain gradient plasticity. J. Mech. Phys. Solids 45, 1253-1273.

Zbib, H., Aifantis, E., 1989. On the localization and post-localization behaviour of plastic deformation. I, II, III. Res. Mechanica 23 261-277; 279-292; 293-305. 